Article

\title{
Thermal Stability and Fire Properties of Salen and Metallosalens as Fire Retardants in Thermoplastic Polyurethane (TPU)
}

\author{
Aditya Ramgobin ${ }^{1}$, Gaëlle Fontaine ${ }^{1}$, Christophe Penverne ${ }^{2}$ and Serge Bourbigot ${ }^{1, *}$ \\ 1 UMR 8207-UMET_Unité Matériaux et Transformations, University of Lille, Ecole Nationale Supérieure de \\ Chimie Lille, F-59652 Villeneuve d'Ascq, France; aa.ramgobin@ed.univ-lille1.fr (A.R.); \\ gaelle.fontaine@ensc-lille.fr (G.F.) \\ 2 USR 3290-MSAP-Miniaturisation pour la Synthèse, l'Analyse et la Protéomique, University of Lille, \\ F-59652 Villeneuve d'Ascq, France; christophe.penverne@univ-lille1.fr \\ * Correspondence: serge.bourbigot@ensc-lille.fr; Tel.: +33-320-434-888
}

Academic Editor: De-Yi Wang

Received: 24 April 2017; Accepted: 12 June 2017; Published: 17 June 2017

\begin{abstract}
This study deals with the synthesis and evaluation of salen based derivatives as fire retardants in thermoplastic polyurethane. Salens, hydroxysalens and their first row transition metal complexes (salen-M) were synthesized (Copper, Manganese, Nickel and Zinc). They were then incorporated in thermoplastic polyurethane (TPU) with a loading as low as 10:1 weight ratio. The thermal stability as well as the fire properties of the formulations were evaluated. Thermogravimetric analysis (TGA) showed that different coordination metals on the salen could induce different decomposition pathways when mixed with TPU. The Pyrolysis Combustion Flow Calorimetry (PCFC) results showed that some M-salen have the ability to significantly decrease the peak heat release rate ( $-61 \%$ compared to neat TPU) and total heat released ( $-63 \%$ compared to neat TPU) when formulated at 10:1 wt \% ratio in TPU. Mass Loss Cone Calorimetry (MLC) results have shown that some additives (salen-Cu and salen-Mn) exhibit very promising performance and they are good candidates as flame-retardants for TPU.
\end{abstract}

Keywords: salen; metallosalen; thermoplastic polyurethane; fire retardants; PCFC; thermogravimetric analysis; Mass Loss Cone Calorimetry

\section{Introduction}

Flame-retardants (FR) are a wide range of substances that are used as additives in a plethora of polymeric materials with the aim of inhibiting, suppressing or delaying ignition in order to prevent the spread of fire [1]. The market today consists of a large variety of FR to contribute to general safety in many applications.

The largest selling inorganic flame retardant by weight is presently aluminum tri-hydroxide (ATH). It is used as a filler in a wide range of elastomers, thermoplastics, and thermosetting resins processed at low temperatures $\left(<200{ }^{\circ} \mathrm{C}\right)$. ATH requires a high loading $(40-60 \mathrm{wt} \%)$ in order to meet acceptable fire properties. The addition of promoters in the polymeric systems to decrease filler proportions is one of the sought out routes to increase the efficiency of such FRs [2]. On the other hand, the most effective, generally applicable commercial fire-retardant systems presently available are based on halogen containing compounds. However, concerns regarding halogenated FR have caused a decline in the demand in the FR market. As a result, halogen free alternatives are being sought in this field [3]. Another type of FR that has gained popularity over the past decades is phosphorus based ones. Indeed, FR research as well as the market on phosphorus-based FR have proliferated partly due 
to the aforementioned increased demand in halogen-free FRs. However, despite the wide array of possibilities that can be exploited with such FRs, there are some issues regarding them. For instance, most inorganic phosphorus based FR additives require very high loadings ( $>30 \mathrm{wt} \%$ ) in order to have a significant effect [4]. This high a loading can have adverse effects on the physical and mechanical properties of the material [1]. Moreover, because phosphorus-based FR may, in some cases, release phosphoric acid, corrosion-related issues of such additives arise [5].

In order to tackle the aforementioned issues, a new class of fire retardant showing promising preliminary results has been developed. The work performed at the laboratory deals with the study of different classes of FR. Indeed, with the aim of developing novel FRs with high efficiency and low toxicity, one such class of FR that was developed concerns $N, N^{\prime}$-(bis salicylidene)ethylenediamine (salen, hereafter called s1) and $N, N^{\prime}$-bis(4-hydroxysalicylidene)ethylenediamine (salen $(\mathrm{OH})_{2}$, hereafter called s2) as well as their copper (II) complexes.

Salens have been extensively studied in different fields, namely, as a catalyst in asymmetric synthesis [6]. Metallosalens have shown to be effective catalysts, which induced the cleavage of DNA [7]. Salens and hydroxysalens have also shown promise in the field of enantioselective epoxidation [8].

They have proved to exhibit highly interesting fire properties in thermoplastic polyurethane (TPU) [9]. Previous studies have shown that s1 has the potential of acting as FR in TPU with loadings as low as $10 \mathrm{wt} \%$ [10]. Previous studies on salens have thoroughly investigated the decomposition mechanism of s2 [11]. It was demonstrated that, under high temperature conditions, s2 has the ability to undergo a polycondensation reaction and crosslink, forming a thermally stable residue; however, s1 did not exhibit this behavior [10]. For this reason, despite the fact that they both exhibit interesting fire properties, it is believed that $\mathbf{s} \mathbf{1}$ and $\mathbf{s} \mathbf{2}$ have different modes of fire retardation. In addition, the complexation of $\mathbf{s} 2$ with copper revealed that degradation temperature of copper-salen complex increases. Indeed, the presence of copper ion as a metallosalen additive in TPU has been shown to enhance the fire properties of TPU/salen formulation. The fire retardant mechanism is reported in an unpublished work where the salen-Cu complex has been shown to work synergistically with the thermal degradation of TPU. It has been reported that metal ions can coordinate to a fragmented section of TPU, keeping it in a condensed phase, and action of the radical formed is also shown [12].

In the pursuit of our effort for developing new FRs, and, in particular, salen-based FRs, the effect of the metallic cation complexed in salens was investigated. Indeed, the choice of the cation should influence the degradation mechanism of the FR as well as its fire properties and provides a promising route to designing new FR. Salen had previously been used in thermoplastic polyurethane (TPU). Therefore, the focus of this work concerns the screening of metallosalen (salen metal complexes, M-salen) in order to determine their thermal stability and investigate their efficiency as FRs in the same polymer. Should an enhanced stability with similar fire properties arise, these FRs would have the potential for wider applicability in polymeric materials with higher processing temperatures.

\section{Results and Discussion}

\subsection{Synthesis and Characterization}

\subsubsection{Salen Ligand}

The salen ligand s1 (Figure 1a) and salen $(\mathrm{OH})_{2}$ s2 (Figure 1b), which are the products of the condensation reaction between the corresponding salicylaldehyde and ethylenediamine, were synthesized using a very simple procedure previously described [9]. The reaction yields were particularly high with $94 \%$ for s1 and $96 \%$ for s2.

The ligands were characterized by nuclear magnetic resonance (NMR) spectroscopy $\left({ }^{1} \mathrm{H},{ }^{13} \mathrm{C}\right.$ and heteronuclear coupling) as well as Infra Red (IR) spectroscopy. 


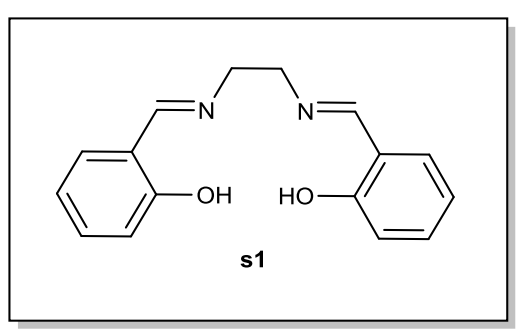

(a)

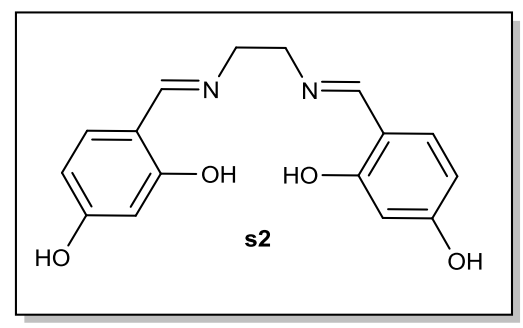

(b)

Figure 1. Structures of (a) the base salen ligand s1 and (b) and salen $(\mathrm{OH})_{2}, \mathbf{s 2}$.

\subsubsection{Salen Complexes}

A range of salen and salen $(\mathrm{OH})_{2}$ complexes (Figure 2) were synthesized. Synthetic methods had to be adapted with respect to the sensitivity of the reactants that were used. The metals that were used were limited to first row earth abundant metals [13] and zinc. Most of the complexes afforded a relatively acceptable yield ranging from $40 \%$ to $80 \%$. However, due to its high air sensitivity, s1-Mn was not synthesized.

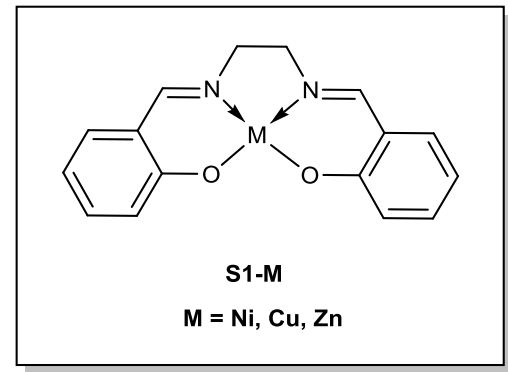

(a)

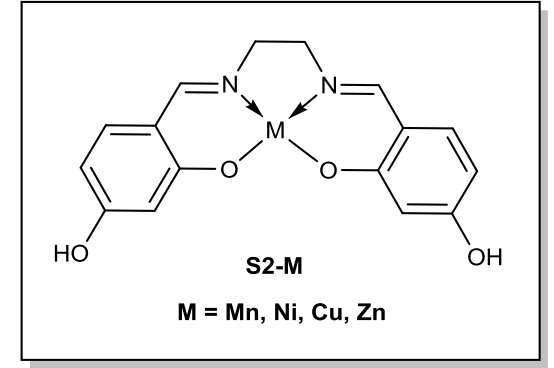

(b)

Figure 2. (a) Panel of metallosalen (labelled s1-M); (b) different metallosalen $(\mathrm{OH})_{2}$ (labelled s2-M).

Most of the complexes were characterized by NMR techniques $\left({ }^{1} \mathrm{H}\right.$ and $\left.{ }^{13} \mathrm{C}\right)$. However, some of the metal ions that were used for the complexation reaction were highly paramagnetic, making their identification via conventional NMR pulse programs impossible. Therefore, IR spectroscopy and mass spectroscopy were also performed to characterize them.

\subsection{Thermal Stability}

\subsubsection{Thermogravimetric Analysis of $\mathbf{s} \mathbf{1}, \mathbf{s} \mathbf{2}$ and Their Complexes}

The thermo-oxidative and pyrolytic decomposition of salens $\mathbf{s} 1$ and $\mathbf{s} 2$ and their corresponding complexes were studied (Figures 3 and 4, Tables 1 and 2). It can be observed that the presence of a coordination metal greatly influences the thermal stability of the substance whatever the atmosphere (nitrogen or in air). A significant increase in the degradation temperature is observed when comparing the thermogravimetric analysis (TGA) plots of the different complexes to the beginning of the decomposition temperature of the base salens s1 and s2. Indeed, while the TG plot shows that s1 starts to degrade at $295^{\circ} \mathrm{C}$, those of the complexes show a decomposition temperature that is at least $45^{\circ} \mathrm{C}$ higher (in the case of s1-Cu). Similarly, in the case of $\mathbf{s} 2$ (decomposition temperature of $171^{\circ} \mathrm{C}$ ), the TGA plots of $\mathbf{s} 2$ complexes show that they start decomposing at a temperature that is at least $108^{\circ} \mathrm{C}$ higher. This enhanced thermal stability may be explained by the strong interaction between the metal ion and the chelating ligand with the oxygen donor atoms. The residual mass at $800{ }^{\circ} \mathrm{C}$ are also significantly higher when the salens are chelated with a metal. 


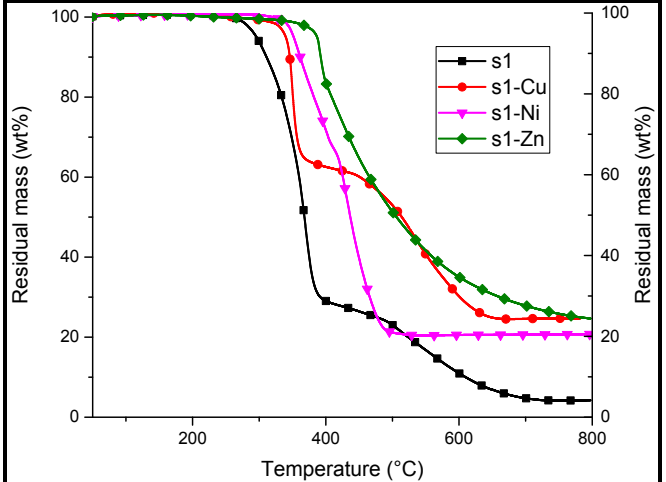

(a)

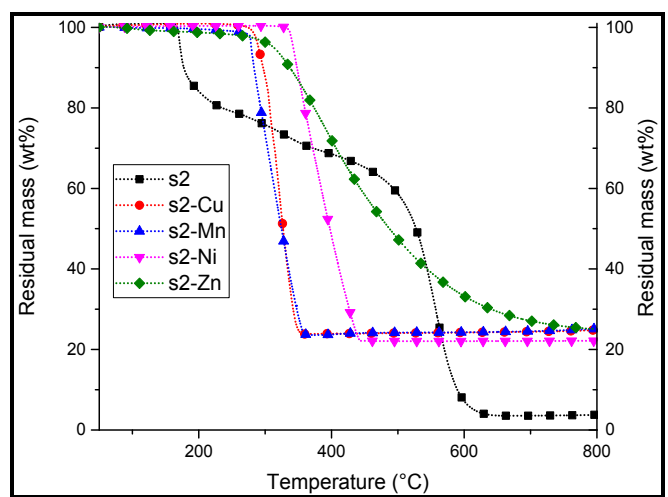

(b)

Figure 3. Thermogravimetric analyses (TGA) at a heating rate of $10^{\circ} \mathrm{C} / \mathrm{min}$ in air of salen (a) black: s1; salen complexes: red: s1-Cu; magenta: s1-Ni and dark green: s1-Zn and $(\mathbf{b}) \operatorname{salen}(\mathrm{OH})_{2}$ : gray: s2, salen $(\mathrm{OH})_{2}$ complexes: orange: s2-Cu; lime: s2-Mn; indigo: s2-Ni, violet: s2-Zn.

In air, s1 undergoes a sharp first degradation step at $295^{\circ} \mathrm{C}$ with a mass loss corresponding to about $65 \%$ of the initial mass (at $400{ }^{\circ} \mathrm{C}$ ). After this step, there is a slow decrease in residual mass until a stable final residue is reached at about $700{ }^{\circ} \mathrm{C}$. This behavior may be attributed to the formation of a transient char that is relatively stable, which undergoes slow decomposition compared to the rate of initial degradation.

Similar thermal behaviors are observed when $\mathbf{s} \mathbf{1}$ is complexed with the different metals (data summarized in Table 1). The presence of a coordination metal chelated to the salen ligand increases its thermal stability. Indeed, the initial degradation temperatures increase significantly when there is a metal attached to the salen framework. In the case of $\mathbf{s 1 - Z n}$, the initial degradation temperature is as high as $386^{\circ} \mathrm{C}\left(+91{ }^{\circ} \mathrm{C}\right.$ as compared to that for s1). In terms of the first degradation step, the TGA curve of s1-Cu shows a sharp mass loss at $340^{\circ} \mathrm{C}$, which is shortly followed by another decomposition step at around $400{ }^{\circ} \mathrm{C}$. This behavior seems to be similar to the decomposition of s1, albeit occurring at a higher temperature.

However, s1-Zn undergoes slow, continuous degradation starting at about $400{ }^{\circ} \mathrm{C}$, suggesting that there is the formation of a transient char right after the start of the decomposition. This is observed during the mass loss cone calorimetry (MLC) test (discussed later), whereby the char formation is observed. s1-Ni decomposes with two successive steps occurring at around $367^{\circ} \mathrm{C}$ (corresponding to a mass loss of $34 \mathrm{wt} \%$ ) and $423{ }^{\circ} \mathrm{C}$ (with a mass loss of $46 \%$ ).

Table 1. Thermogravimetric data of salen, and their complexes under thermo-oxidative atmosphere at $10{ }^{\circ} \mathrm{C} / \mathrm{min}$.

\begin{tabular}{|c|c|c|c|c|c|c|c|c|c|c|}
\hline \multirow[b]{2}{*}{$\begin{array}{c}\text { Metal } \\
\text { Complex }\end{array}$} & \multicolumn{5}{|c|}{ s1 } & \multicolumn{5}{|c|}{ s2 } \\
\hline & $\begin{array}{l}T_{5 \%} \\
\left({ }^{\circ} \mathrm{C}\right)\end{array}$ & $\begin{array}{c}T_{\mathrm{MAX} 1} \\
\left({ }^{\circ} \mathrm{C}\right)\end{array}$ & $\begin{array}{c}T_{\text {MAX2 }} \\
\left({ }^{\circ} \mathrm{C}\right)\end{array}$ & $\begin{array}{c}M L R \\
\left(\% /{ }^{\circ} \mathrm{C}\right)\end{array}$ & $\begin{array}{l}\text { Res } \\
(\%)\end{array}$ & $\begin{array}{l}T_{5 \%} \\
\left({ }^{\circ} \mathrm{C}\right)\end{array}$ & $\begin{array}{c}T_{\text {MAX }} \\
\left({ }^{\circ} \mathrm{C}\right)\end{array}$ & $\begin{array}{c}T_{\mathrm{MAX} 2} \\
\left({ }^{\circ} \mathrm{C}\right)\end{array}$ & $\begin{array}{l}\text { MLR1 } \\
\left(\% /{ }^{\circ} \mathrm{C}\right)\end{array}$ & $\begin{array}{l}\text { Res } \\
(\%)\end{array}$ \\
\hline- & 295 & 369 & - & $1.5^{1}$ & 4 & 171 & $171^{1}$ & 556 & $1.3^{1}$ & 4 \\
\hline $\mathrm{Cu}$ & 340 & 349 & 325 & $2.4^{1}$ & 25 & 290 & $285^{1}$ & 325 & $1.3^{1}$ & 23 \\
\hline $\mathrm{Mn}$ & $\mathrm{N} / \mathrm{A}$ & $\mathrm{N} / \mathrm{A}$ & $\mathrm{N} / \mathrm{A}$ & $\mathrm{N} / \mathrm{A}$ & N/A & 279 & $279^{1}$ & - & $1.5^{1}$ & 25 \\
\hline $\mathrm{Ni}$ & 351 & 428 & 368 & $0.9^{2}$ & 20 & 342 & $347^{1}$ & - & $0.9^{1}$ & 22 \\
\hline $\mathrm{Zn}$ & 386 & 392 & - & $1.1^{1}$ & 25 & 313 & $386^{1}$ & - & $1.4^{1}$ & 25 \\
\hline
\end{tabular}

${ }^{1}$ MLR $_{\text {Max }}$ occurs at the first step of the degradation; ${ }^{2}$ MLR $_{\text {MAX }}$ occurs at the second step of the degradation. MLR: Mass loss rate; Res: Residual mass.

In the case of s2 and s2-M, initial degradation occurs at lower temperatures than does that for s1 and s1-M. The thermal decomposition of $\mathbf{s} 2$ occurs in two major steps similar to that for $\mathbf{s 1}$. The first step corresponds to a lower mass loss and takes place at a lower temperature $\left(170^{\circ} \mathrm{C}\right)$. 
From previous studies of the thermal degradation of $\mathbf{s} \mathbf{2}$, it is known that $\mathbf{s} \mathbf{2}$ has the ability to form a phenolic resin. This occurs via a polycondensation reaction involving the formation of methylene bridges sandwiched between aromatic rings. It was also shown that water is evolved at around 190 and $200{ }^{\circ} \mathrm{C}$. This suggests that there is some form of condensation reaction occurring. This could explain the relatively low temperature degradation of $\mathbf{s} 2\left(170^{\circ} \mathrm{C}\right)$ that corresponds to degradation of s2, which forms fragments that subsequently react to form a transient char, which is assumed to be a thermally stable polycondensed phenolic resin [10]. The degradation of this char $\left(556{ }^{\circ} \mathrm{C}\right)$ occurs at a higher temperature than does $\mathbf{s} 1\left(500^{\circ} \mathrm{C}\right)$.

On the other hand, the TGA plots of s2-M complexes, except that for s2-Zn, show a single sharp decomposition at around $300-400{ }^{\circ} \mathrm{C}$, followed by the formation of a highly stable residue. This is attributed to the metal oxide of the cation used. s2-Zn has a different decomposition behavior as compared to the other s2-M complexes. Indeed, as for $\mathbf{~} \mathbf{1 - Z n}$, a slow, continuous decomposition of the complex as early as at the start of the degradation is observed in the TGA plot. Despite the residual mass being relatively close to that for the other complexes, it suggests that there is a different mode of decomposition with zinc.

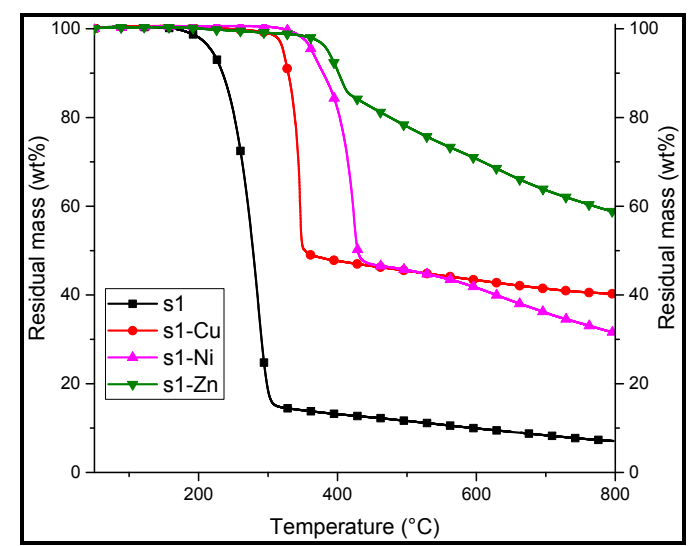

(a)

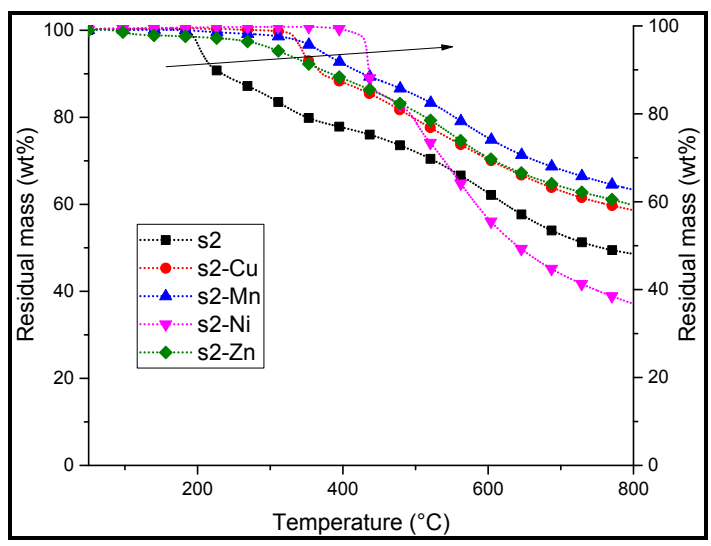

(b)

Figure 4. Thermogravimetric analyses (TGA) at a heating rate of $10^{\circ} \mathrm{C} / \mathrm{min}$ under nitrogen of (a) salen s1 (black) and salen complexes: s1-Cu (red), s1-Ni (magenta), s1-Zn (green) and (b) salen $(\mathrm{OH})_{2}$, s2 (black), salen $(\mathrm{OH})_{2}$ complexes: s2-Cu (red), s2-Mn (blue), s2-Ni (magenta) and s2-Zn (green). The black arrow on figure $4 \mathrm{~b}$ shows the increase in thermal stability of the complexes.

Different decomposition behaviors are observed when the TGA are carried out under pyrolysis conditions (Table 2). Indeed, s1 degrades firstly with a sharp step that begins at $219{ }^{\circ} \mathrm{C}\left(T_{5 \%}\right)$. After the first degradation, there is a slow, linear decrease in residual mass (from $15 \mathrm{wt} \%$ at $309^{\circ} \mathrm{C}$ to $7 \mathrm{wt} \%$ at $\left.800^{\circ} \mathrm{C}\right)$.

Similar results are observed when the $\mathbf{s} 1$ is complexed with the different metal cations where the decomposition of the s1-M complexes occur in a single step. s1-Cu degrades at $322{ }^{\circ} \mathrm{C}$ and leaves a residual mass of $40 \mathrm{wt} \%$. It has the lowest degradation temperature among the complexes. s1-Ni decomposes at a higher temperature $\left(363{ }^{\circ} \mathrm{C}\right.$ as compared to s1-Cu) with a lower residual mass (31 wt \%), and s1-Zn has the highest thermal stability with a decomposition temperature of $383^{\circ} \mathrm{C}$. It also has the highest remaining mass of $58 \mathrm{wt} \%$ at $800{ }^{\circ} \mathrm{C}$.

As it was expected from the above discussion, the degradation temperatures depend on the coordination metal used (degradation temperature increased from 290 to $386{ }^{\circ} \mathrm{C}$ in the case of $\mathbf{s 1 - Z n}$ ). Moreover, there is a significantly higher residual mass compared to the neat ligand at the end of the measurement $\left(\right.$ at $800^{\circ} \mathrm{C}$ ), strengthening the hypothesis that the metal complex causes the formation of a more stable char. Most samples have a relatively high residual mass at $800{ }^{\circ} \mathrm{C}\left(M_{\text {res }}>30 \mathrm{wt} \%\right)$. 
Table 2. Thermogravimetric data for the decomposition of salen in an inert atmosphere at $10^{\circ} \mathrm{C} / \mathrm{min}$.

\begin{tabular}{|c|c|c|c|c|c|c|c|c|c|c|c|}
\hline \multirow{2}{*}{ Sample } & \multicolumn{5}{|c|}{ s1 } & \multirow[b]{2}{*}{ Sample } & \multicolumn{5}{|c|}{ s2 } \\
\hline & $\begin{array}{l}T_{5 \%} \\
\left({ }^{\circ} \mathrm{C}\right) \\
\end{array}$ & $\begin{array}{c}T_{\text {MAX }} \\
\left({ }^{\circ} \mathrm{C}\right)\end{array}$ & $T_{\text {MAX2 }}$ & $\begin{array}{c}M L R \\
\left(\% /{ }^{\circ} \mathrm{C}\right)\end{array}$ & $\begin{array}{l}\text { Res } \\
(\%)\end{array}$ & & $\begin{array}{l}T_{5 \%} \\
\left({ }^{\circ} \mathrm{C}\right)\end{array}$ & $\begin{array}{c}T_{\text {MAX1 } 1} \\
\left({ }^{\circ} \mathrm{C}\right)\end{array}$ & $\begin{array}{c}T_{\mathrm{MAX} 2} \\
\left({ }^{\circ} \mathrm{C}\right)\end{array}$ & $\begin{array}{l}M L R \\
\left(\% /{ }^{\circ} \mathrm{C}\right)\end{array}$ & $\begin{array}{l}\text { Res } \\
(\%)\end{array}$ \\
\hline- & 219 & $287^{1}$ & - & $1.7^{1}$ & 7 & - & 206 & & 200 & $0.1^{1}$ & 49 \\
\hline s1-Cu & 322 & 328 & 345 & $4.8^{2}$ & 40 & $\mathrm{~s} 2-\mathrm{Cu}$ & 345 & 334 & 345 & $0.26^{2}$ & 59 \\
\hline s1-Mn & $\mathrm{N} / \mathrm{A}$ & $\mathrm{N} / \mathrm{A}$ & $\mathrm{N} / \mathrm{A}$ & $\mathrm{N} / \mathrm{A}$ & $\mathrm{N} / \mathrm{A}$ & s2-Mn & 371 & 375 & 550 & $0.1^{2}$ & 63 \\
\hline s1-Ni & 363 & 368 & 424 & $1.7^{2}$ & 31 & $\mathrm{~s} 2-\mathrm{Ni}$ & 432 & 435 & 502 & $1.6^{1}$ & 37 \\
\hline s1-Zn & 386 & $405^{1}$ & - & $0.4^{1}$ & 58 & s2-Zn & 314 & 330 & 555 & $0.1^{2}$ & 60 \\
\hline
\end{tabular}

$T_{5 \%}$ corresponds to the temperature at which a mass loss of $5 \mathrm{wt} \%$ is recorded. ${ }^{1}$ MLR $\mathrm{R}_{\text {Max }}$ occurs at the first step of the degradation; ${ }^{2}$ MLR $_{\text {MAX }}$ occurs at the second step of the degradation.

In the case of $\mathbf{s} 2$ under pyrolytic conditions, the decomposition also starts at a lower temperature than s1. However, it occurs at a much lower rate and corresponds to a lower mass loss (about $10 \mathrm{wt} \%$ instead of about $85 \mathrm{wt} \%$ ). It is followed by a slow degradation with respect to temperature, yielding a residue of around $49 \mathrm{wt} \%$ (about seven times more than s1).

Similar behavior is observed by most salen $(\mathrm{OH})_{2}$ complexes, s2-M. Their TGA plots show a sharp mass loss at $350-400{ }^{\circ} \mathrm{C}$ followed by slow degradation, except for s2-Mn, with which the TGA plot shows only a continuous, slow rate of degradation. This suggests that there might be a different mode of degradation with these complexes as compared to the other $\mathbf{s} 2$ complexes.

Table 3 illustrates the calculated weight percent of the metal oxides with the assumption that the decomposition of the complex led only to the formation of the metal oxides. Under inert atmosphere, the remaining mass at $800^{\circ} \mathrm{C}$ is significantly higher than the calculated weight percent of the metal oxide. This suggests that there might be the formation of a thermally stable structure. The general trend suggests that the s2-M complexes have a slightly higher residual mass than the s1-M complexes.

This enhancement of the thermal stability was already reported [11]. The s2 ligand showed the ability to form a stable cross-linked phenolic resin under thermal stress. In this study, it has been shown that the presence of a metal in the $\mathbf{s} 2$ ligand keeps the FR's ability to form a stable material at high temperatures. The chelation by different metals increases the degradation temperature of the FR, thus, extending their applicability to polymers with higher processing temperatures.

Table 3. wt \% of metal oxides as compared to the actual remaining masses of the complexes after thermogravimetric analysis (TGA) in inert and thermo-oxidative atmosphere.

\begin{tabular}{|c|c|c|c|c|c|c|}
\hline \multirow{2}{*}{ Sample } & \multicolumn{3}{|c|}{ s1 } & \multicolumn{3}{|c|}{ s2 } \\
\hline & MO (\%) ${ }^{T}$ & $\operatorname{Res}(\%)\left(\mathrm{N}_{2}\right)$ & Res (\%) (Air) & MO (\%) ${ }^{1}$ & $\operatorname{Res}(\%)\left(\mathrm{N}_{2}\right)$ & Res (\%) (Air) \\
\hline $\mathrm{Cu}$ & 25 & 40 & 25 & 22 & 59 & 23 \\
\hline $\mathrm{Mn}$ & $\mathrm{N} / \mathrm{A}$ & $\mathrm{N} / \mathrm{A}$ & $\mathrm{N} / \mathrm{A}$ & 20 & 63 & 25 \\
\hline $\mathrm{Ni}$ & 23 & 31 & 20 & 21 & 37 & 22 \\
\hline $\mathrm{Zn}$ & 24 & 58 & 25 & 22 & 60 & 25 \\
\hline
\end{tabular}

${ }^{1}$ weight percent of metal oxides with respect to the complex. MO: Metal Oxide.

The results show that the salen and metallosalen complexes would remain stable at the processing temperature of TPU $\left(190^{\circ} \mathrm{C}\right)$.

\subsubsection{Thermogravimetric Analysis (TGA) of TPU/Salen Formulations}

TPU formulations containing 10:1 weight ratio of TPU/additives (salen and their complexes) were subjected to TGA analyses in nitrogen (Figure 5) and in air (Figure 6). The aim was to evaluate the effect (stabilization or destabilization) of the FRs compared to the neat polymer (Tables 4 and 5).

In thermo-oxidative atmosphere, the TGA curves of all formulations including neat TPU show a two-step decomposition process. All of the metal complexes based formulations show a much lower decomposition temperature at $5 \mathrm{wt} \%$ ranging from 290 to $298^{\circ} \mathrm{C}$ as compared to neat TPU $\left(312{ }^{\circ} \mathrm{C}\right)$. 
The second decomposition step corresponds to the degradation of a transient char, which decomposes at a higher temperature than the first decomposition temperature. In the case of TPU/s1-Zn, the TGA curve shows a first step that occurs with a very high mass loss rate $(2.3 \mathrm{wt} \% / \mathrm{s}$ as compared to $1.5 \mathrm{wt} \% / \mathrm{s}$ for neat TPU) and a second step occurring at a much higher temperature than the others $\left(450^{\circ} \mathrm{C}\right)$. Based on previously reported work, it suggests that there is formation of a stable compound after the first step of the decomposition. This compound remains relatively stable at high temperatures. Such a behavior is known to occur with the dihydroxy(salen) when it is subjected to a high temperature stress. Indeed, dihydroxysalen has the ability to form cross-linked polymeric structures when subjected to thermal stress [11]. With the exception of TPU/s1-Ni, there is a slight increase in residual mass of the formulations.

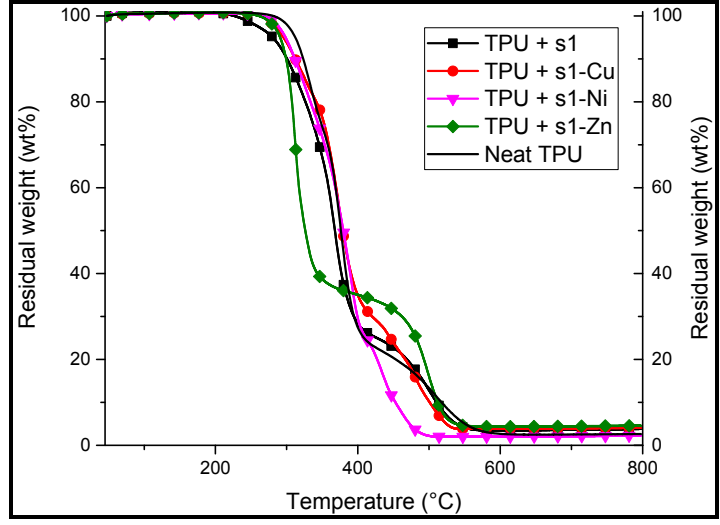

(a)

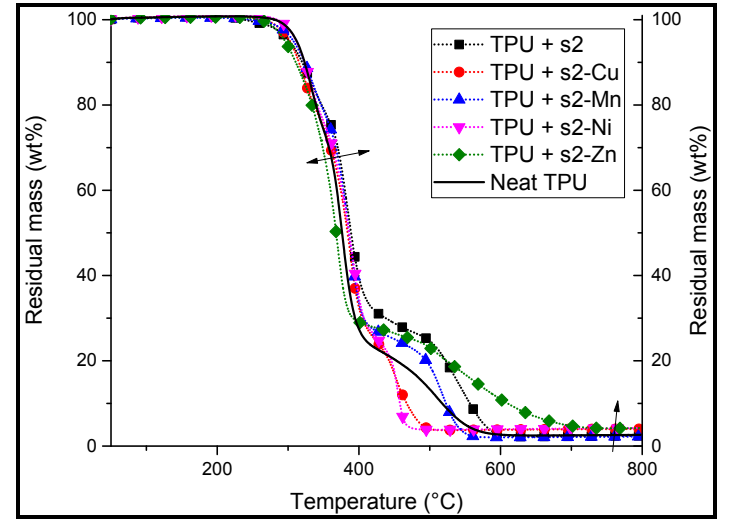

(b)

Figure 5. TGA analyses under thermo-oxidative conditions (air) of TPU formulations with 10:1 wt \% ratio of (a) s1 and its corresponding complexes; (b) s2 and its corresponding complexes. The double ended arrow shows the small variation in the decomposition temperatures of the formulations.

With the exception of zinc-based formulations, the TGA curves of TPU/s2 formulations show similar behaviors as TPU/s1 formulations under air. The TGA plots of all the formulations exhibit a two-stage decomposition. The first degradation temperatures range from 295 to $311^{\circ} \mathrm{C}$, with TPU/s2-Zn starting its degradation at the lowest $\left(295^{\circ} \mathrm{C}\right)$ temperature and that of s2-Ni at the highest $\left(311^{\circ} \mathrm{C}\right)$. The mass loss rates (MLR) are also relatively close to the MLR of neat TPU. However, in the case of $\mathbf{s 2}$, s2-Mn and $\mathbf{s 2 - Z n}$, the TGA plots show that the second degradation of the formulations occurs at a higher temperature than the other formulations $\left(>500^{\circ} \mathrm{C}\right)$. It is assigned to the ability of s2 to form thermally stable resins at high temperatures [9]. The presence of these metal ions in the complex may catalyze this mechanism of resin formation and polycondensation making a greater amount of the thermally stable, cross-linked structure [11].

Table 4. Thermogravimetric data for TPU/salen or salen complexes (10:1 weight ratio) formulation in air at $10{ }^{\circ} \mathrm{C} / \mathrm{min}$.

\begin{tabular}{|c|c|c|c|c|c|c|c|c|c|c|c|}
\hline \multirow[b]{2}{*}{ Sample } & \multicolumn{5}{|c|}{ s1 } & \multirow[b]{2}{*}{ Sample } & \multicolumn{5}{|c|}{ s2 } \\
\hline & $\begin{array}{l}T_{5 \%} \\
\left({ }^{\circ} \mathrm{C}\right)\end{array}$ & $\begin{array}{c}T_{\text {MAX1 }} \\
\left({ }^{\circ} \mathrm{C}\right)\end{array}$ & $\begin{array}{c}T_{\text {MAX2 }} \\
\left({ }^{\circ} \mathrm{C}\right)\end{array}$ & $\begin{array}{c}M L R \\
\left(\% /{ }^{\circ} \mathrm{C}\right)\end{array}$ & $\begin{array}{l}\text { Res } \\
(\%)\end{array}$ & & $\begin{array}{l}T_{5 \%} \\
\left({ }^{\circ} \mathrm{C}\right)\end{array}$ & $\begin{array}{c}T_{\text {MAX1 }} \\
\left({ }^{\circ} \mathrm{C}\right)\end{array}$ & $\begin{array}{c}T_{\mathrm{MAX} 2} \\
\left({ }^{\circ} \mathrm{C}\right)\end{array}$ & $\begin{array}{c}M L R \\
\left(\% /{ }^{\circ} \mathrm{C}\right)\end{array}$ & $\begin{array}{l}\text { Res } \\
(\%)\end{array}$ \\
\hline Neat TPU & 312 & 331 & 376 & $1.5^{2}$ & 3 & Neat TPU & 312 & 331 & $376^{2}$ & $1.5^{2}$ & 3 \\
\hline TPU/s1 & 280 & 367 & 505 & $1.2^{1}$ & 4 & TPU/s2 & 303 & 335 & $384^{2}$ & $1.2^{2}$ & 2 \\
\hline TPU/s1-Cu & 295 & 316 & 374 & $1.1^{2}$ & 4 & TPU/s2-Cu & 302 & 317 & $370^{2}$ & $1.2^{2}$ & 4 \\
\hline TPU/s1-Mn & $\mathrm{N} / \mathrm{A}$ & $\mathrm{N} / \mathrm{A}$ & $\mathrm{N} / \mathrm{A}$ & $\mathrm{N} / \mathrm{A}$ & $\mathrm{N} / \mathrm{A}$ & TPU/s2-Mn & 307 & 332 & $388^{2}$ & $1.3^{2}$ & 2 \\
\hline TPU/s1-Ni & 298 & 328 & 391 & $1.1^{2}$ & 2 & TPU/s2-Ni & 311 & 332 & $378^{2}$ & $1.4^{2}$ & 4 \\
\hline TPU/s1-Zn & 290 & 312 & 501 & $2.3^{1}$ & 5 & TPU/s2-Zn & 295 & 310 & $371^{2}$ & $1.4^{2}$ & 5 \\
\hline
\end{tabular}

${ }^{1}$ MLR $_{\text {Max }}$ occurs at the first step of the degradation; ${ }^{2}$ MLR $_{\text {MAX }}$ occurs at the second step of the degradation. 
In pyrolytic conditions (Figure 6 and Table 5), TPU/s1 shows a slight decrease of the thermal stability compared to that of neat TPU and TPU/s1-M complexes. Indeed, the decomposition of the TPU / s1 starts at $287^{\circ} \mathrm{C}$ whereas that of neat TPU and the other formulations show an initial mass loss $(95 \mathrm{wt} \%)$ at a higher temperature of around $300^{\circ} \mathrm{C}$.

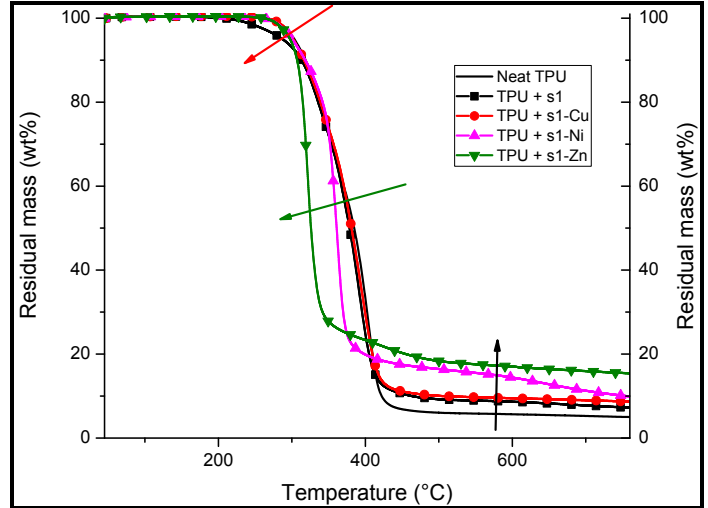

(a)

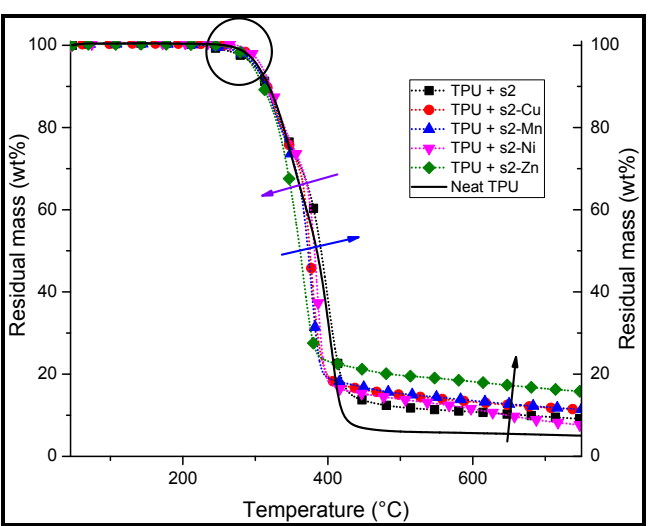

(b)

Figure 6. TGA analyses under inert atmosphere $\left(\mathrm{N}_{2}\right)$ of TPU formulations with 10:1 wt \% ratio of (a) s1 and its corresponding complexes; (b) s2 and its corresponding complexes. The red arrow shows the general trend of the decomposition temperature of the additives. The green arrow shows the relatively low temperature at which the $\mathbf{s 1 - Z n}$ decomposes as compared to the rest of the formulations. The black arrows show increased residual masses at the second stage of the decomposition. The violet and blue arrows show the increase or decrease in the mass loss rates. The circle shows the initial decomposition stage of the formulations.

However, despite showing a higher decomposition temperature than TPU/s1, the TGA plots of both TPU/s1-Ni and TPU/s1-Zn show that they have a first decomposition stage that is steeper than the other formulations (green arrow, Figure 6a). They also correspond to a lower mass loss than do the other s1-M formulations. Moreover, all of the formulations show a residual mass at $750{ }^{\circ} \mathrm{C}$ that is higher than that of the neat TPU (black arrow, Figure 6a). This suggests that there is polymer/FR interactions promoting charring.

Table 5. Thermogravimetric data for TPU/salen formulation in pyrolytic conditions at $10^{\circ} \mathrm{C} / \mathrm{min}$.

\begin{tabular}{|c|c|c|c|c|c|c|c|c|c|c|c|}
\hline \multirow[b]{2}{*}{ Sample } & \multicolumn{5}{|c|}{ s1 } & \multirow[b]{2}{*}{ Sample } & \multicolumn{5}{|c|}{$\mathrm{s} 2$} \\
\hline & $\begin{array}{l}T_{5 \%} \\
\left({ }^{\circ} \mathrm{C}\right)\end{array}$ & $\begin{array}{c}T_{\text {MAX1 } 1} \\
\left({ }^{\circ} \mathrm{C}\right)\end{array}$ & $\begin{array}{c}T_{\mathrm{MAX} 2} \\
\left({ }^{\circ} \mathrm{C}\right)\end{array}$ & $\begin{array}{l}M L R \\
\left(\% /{ }^{\circ} \mathrm{C}\right)\end{array}$ & $\begin{array}{l}\text { Res } \\
(\%)\end{array}$ & & $\begin{array}{l}T_{5 \%} \\
\left({ }^{\circ} \mathrm{C}\right)\end{array}$ & $\begin{array}{c}T_{\text {MAX1 } 1} \\
\left({ }^{\circ} \mathrm{C}\right)\end{array}$ & $\begin{array}{c}T_{\mathrm{MAX}} \\
\left({ }^{\circ} \mathrm{C}\right)\end{array}$ & $\begin{array}{l}M L R \\
\left(\% /{ }^{\circ} \mathrm{C}\right)\end{array}$ & $\begin{array}{l}\text { Res } \\
(\%)\end{array}$ \\
\hline $\begin{array}{l}\text { Neat } \\
\text { TPU }\end{array}$ & 303 & 303 & $406^{2}$ & 1.4 & 5 & $\begin{array}{l}\text { Neat } \\
\text { TPU }\end{array}$ & 303 & 354 & $406^{2}$ & 1.4 & 5 \\
\hline s1 & 287 & 347 & $391^{2}$ & 1.2 & 7 & s2 & 298 & 344 & $407^{2}$ & 1.2 & 9 \\
\hline s1-Cu & 301 & 343 & $396^{2}$ & 1.1 & 9 & $\mathrm{~s} 2-\mathrm{Cu}$ & 303 & 324 & $377^{2}$ & 2 & 11 \\
\hline s1-Mn & $\mathrm{N} / \mathrm{A}$ & $\mathrm{N} / \mathrm{A}$ & $\mathrm{N} / \mathrm{A}$ & $\mathrm{N} / \mathrm{A}$ & $\mathrm{N} / \mathrm{A}$ & s2-Mn & 300 & 323 & $376^{2}$ & 1.4 & 11 \\
\hline s1-Ni & 300 & 306 & 363 & $2.7^{2}$ & 10 & s2-Ni & 308 & 327 & $386^{2}$ & 1.4 & 7 \\
\hline s1-Zn & 297 & 323 & - & $2.9^{1}$ & 15 & s2-Zn & 306 & 309 & $366^{2}$ & 1.4 & 15 \\
\hline
\end{tabular}

${ }^{1} \mathrm{MLR}_{\mathrm{Max}}$ occurs at the first step of the degradation; ${ }^{2} \mathrm{MLR}_{\mathrm{MAX}}$ occurs at the second step of the degradation.

TGA of TPU containing s2 and its complexes show that they have similar thermal behavior to neat TPU in terms of the first decomposition temperature under nitrogen (encircled), which begins around $300{ }^{\circ} \mathrm{C}$ for every formulation. Moreover, there is a higher residual mass at $750{ }^{\circ} \mathrm{C}$ for the formulated samples (black arrow, Figure $6 \mathrm{~b}$ ) as compared to that of neat TPU. The TGA curve of $\mathrm{TPU} / \mathbf{s} 2-\mathrm{Zn}$ shows the highest remaining mass at $750{ }^{\circ} \mathrm{C}(15 \mathrm{wt} \%)$. It is noteworthy that the mass of 
the corresponding metal oxide does not account for the totality of the residual mass. The TGA curves of s2-Cu and s2-Ni show higher mass loss rates (MLR) than the other formulations (Table 5).

However, the TGA curve of TPU/s2-Zn shows an apparent single-step decomposition unlike the other formulations, which appear to decompose in two steps, as for neat TPU. The apparent single-step decomposition of TPU/s2-Zn is assigned to the overlapping of the two decomposition stages of the formulation. Indeed, this is made evident on the DTG (derivative thermogravimetric) curve, whereby two distinct steps are observed (curve not shown). The residual masses at $750{ }^{\circ} \mathrm{C}$ are significantly higher for all the s2-complex formulations compared to that of neat TPU (as high as $50 \mathrm{wt} \%$ more in the case of TPU/s2-Zn). This suggests that there is the formation of thermally robust char.

In conclusion, it is observed that 10:1 $\mathrm{wt} \%$ ratio of salen or their complexes in TPU can significantly change the thermal stability of the TPU/salen system. The results suggest increased charring and have shown different mass loss rates (MLRs). This suggests that the mode of decomposition of TPU may be altered simply by mixing it with a small amount $(10: 1 \mathrm{wt} \%$ ratio) of salen or metal-salen complex.

Differential TGA under air is shown in Figure 7. This was undertaken to point out the differences in stabilization/destabilizations brought about by the presence of the different additives. Regarding TPU/s1 and s1-M formulations, a major destabilization is observed in the differential TGA curve. This destabilization is much higher in the case of TPU/s1-Zn, whereby the curve peaks at a value as high as $35 \%$ between 300 and $400{ }^{\circ} \mathrm{C}$. It is followed by a stabilization of around $10 \%$ at high temperatures $\left(400-500{ }^{\circ} \mathrm{C}\right)$. However, with the exception of the TPU/s1-Ni formulation, there is

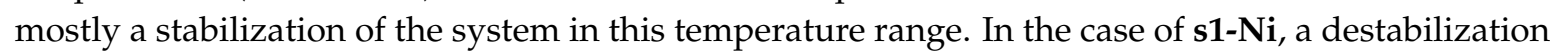
is observed for the whole range of temperatures with a most important one between 400 and $600{ }^{\circ} \mathrm{C}$. TPU/s2 and s2-M formulations behave differently. Indeed, with the exception of TPU/s2-Zn, there appears to be a stabilization of $10-18 \%$ at about $380^{\circ} \mathrm{C}$ in the differential TGA plot. However, the curve corresponding to TPU/s2-Zn shows a destabilization between 280 and $400{ }^{\circ} \mathrm{C}$ followed by a stabilization of $10 \%$ between 400 and $600{ }^{\circ} \mathrm{C}$. It suggests that the interactions could stabilize char at high temperature, and so it should bring benefits in terms of reaction to fire.

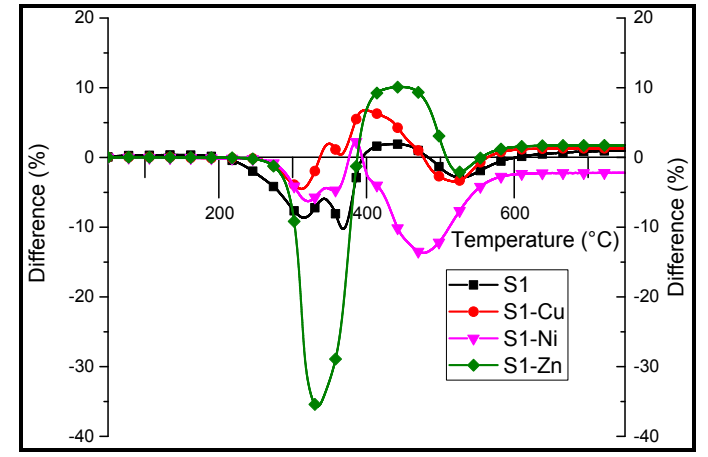

(a)

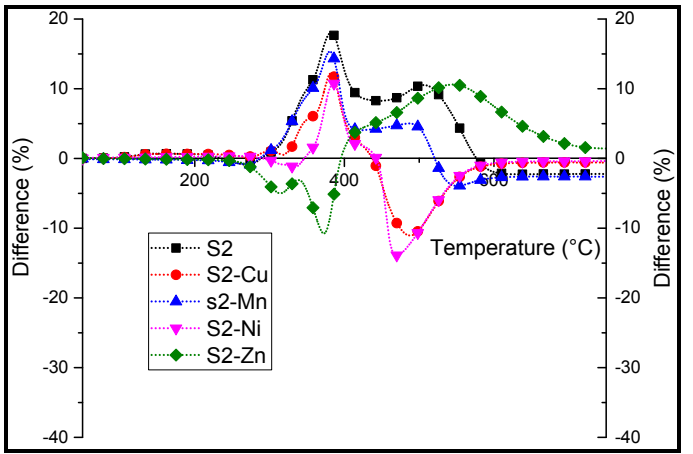

(b)

Figure 7. Differential thermogravimetric analysis (experimental percentage residual mass-calculated percentage residual mass) under thermo-oxidative atmosphere of (a) s1 and the complexes and (b) metallosalen complexes when formulated with TPU at 10:1 wt \% ratio.

\subsection{Pyrolysis Combustion Flow Calorimetry}

Pyrolysis combustion flow calorimetry (PCFC) was carried out on both the flame-retardants and the formulated samples. This evaluation provides us with results regarding the specific heat release rate (HRR), the temperature at which the peak heat release rate (pHRR) occurs as well as the total heat released. The results are summarized in Table 6.

Apart from s1 and s1-Ni, the PCFC curves of every TPU containing s1-based additives show a decrease in the pHRR compared to that of neat TPU. The s1-Zn formulation has the lowest temperature at pHRR (Figure 8a) and it has the lowest total heat released (HR) (Table 6). For the 
other s1-complexes, the pHRR occurs between 410 and $435^{\circ} \mathrm{C}$. The peaks exhibit different widths at half height and have only one maximum. This broadness implies that the combustibles in the tested samples are released at a wide range of temperatures.

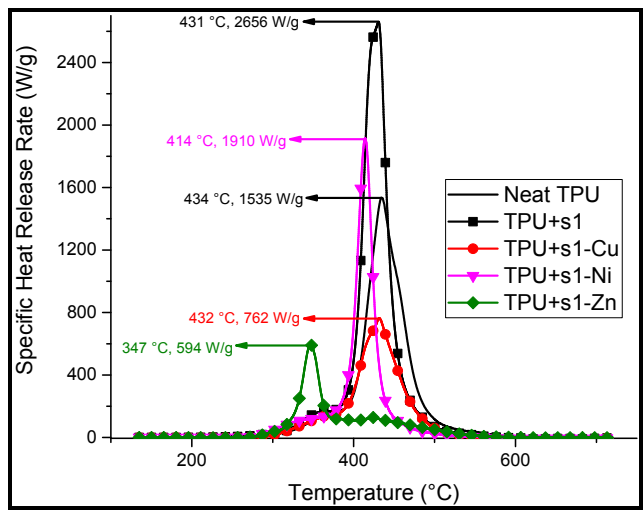

(a)

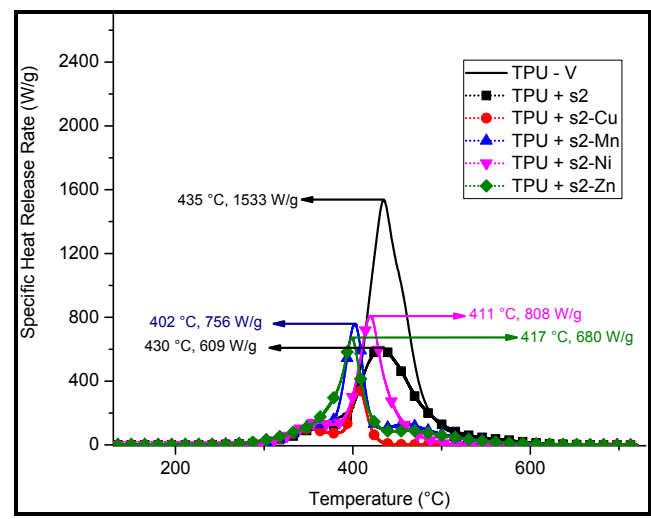

(b)

Figure 8. Corrected pyrolysis combustion flow calorimetry (PCFC) curves of (a) neat TPU, TPU/s1 (10:1 wt \% ratio) and TPU/s1-metal (10:1 wt \% ratio); (b) neat TPU, TPU/s2 (10:1 wt \% ratio) and TPU/s2-metal (10:1 wt \% ratio). The values of the temperature at the peak heat release rate (pHRR) as well as the pHRR are shown by the arrows.

In the case of the formulations containing s2 and its complexes, the PCFC curves of all the samples show a decrease in the pHRR (Figure 8b) and total heat released (Table 6) compared to neat TPU. The lowest pHRR is observed on the PCFC curve of TPU/s1-Cu $(680 \mathrm{~W} / \mathrm{g})$, corresponding to a decrease of $56 \%$ as compared to that of neat TPU. The lowest total heat released (THR) corresponds to the TPU/s2-Mn formulation with $38 \mathrm{~kJ} / \mathrm{g}$ compared to $90 \mathrm{~kJ} / \mathrm{g}$ for neat TPU (-58\%). It appears that additional peaks are observed before and after the pHRR. This multi-peak phenomenon may correlate to the TGA curves of the TPU/s2 and TPU/s2-M (Figure 6b). The two-step decomposition of the TPU/s2 formulations may thus be further inferred from the PCFC results above.

It is worth noting that there is, on average, more than a $50 \%$ decrease in the total heat release rate when the TPU/s2 complexes are tested with TPU in the PCFC.

Table 6. Summary of the peak heat release rate, temperature at peak heat release rate and total heat released for the TPU/salen (90/10) formulations. pHRR: peak heat release rate; THR: total heat released.

\begin{tabular}{cccc}
\hline Sample & pHRR $(\mathbf{W} / \mathbf{g})$ & $\mathbf{T}\left({ }^{\circ} \mathbf{C}\right) @ \mathbf{p H R R}(\mathbf{W} / \mathbf{g})$ & THR $(\mathbf{k J} / \mathbf{g})$ \\
\hline Neat TPU & 1535 & 435 & 90 \\
TPU + s1 & $2659(+73 \%)$ & $431\left(-4^{\circ} \mathrm{C}\right)$ & $116(+29 \%)$ \\
TPU + s1-Cu & $763(-50 \%)$ & $431\left(-4^{\circ} \mathrm{C}\right)$ & $52(-42 \%)$ \\
TPU + s1-Ni & $1910(+24 \%)$ & $414\left(-21^{\circ} \mathrm{C}\right)$ & $65(-28 \%)$ \\
TPU + s1-Zn & $594(-61 \%)$ & $346\left(-89^{\circ} \mathrm{C}\right)$ & $33(-63 \%)$ \\
\hline TPU + s2 & $608(-60 \%)$ & $430\left(-5^{\circ} \mathrm{C}\right)$ & $54(-40 \%)$ \\
TPU + s2-Cu & $680(-56 \%)$ & $417\left(-18^{\circ} \mathrm{C}\right)$ & $42(-53 \%)$ \\
TPU + s2-Mn & $756(-51 \%)$ & $402\left(-33^{\circ} \mathrm{C}\right)$ & $38(-58 \%)$ \\
TPU + s2-Ni & $808(-47 \%)$ & $420\left(-15^{\circ} \mathrm{C}\right)$ & $39(-57 \%)$ \\
TPU + s2-Zn & $1246(-19 \%)$ & $411\left(-24^{\circ} \mathrm{C}\right)$ & $46(-49 \%)$ \\
\hline
\end{tabular}

In order to investigate further the decomposition behavior of the formulations, the PCFC results were correlated to the TGA curves i.e., the specific heat release rate as a function of residual mass. This allowed observing the evolution of the mass of the sample as well as the degree of decomposition at which there is a notable release of combustibles. This led to a representation of the amount of heat released with respect to the remaining mass of the samples (Figure 9). The same scales were used for both sets of curves for comparison purposes. 


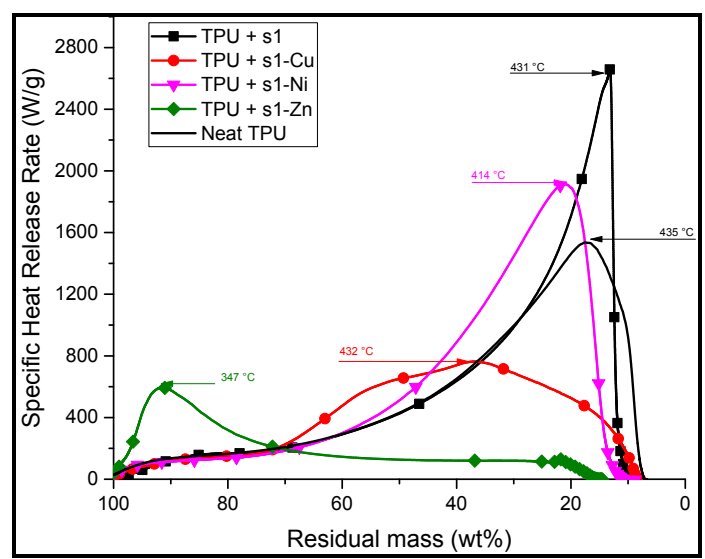

(a)

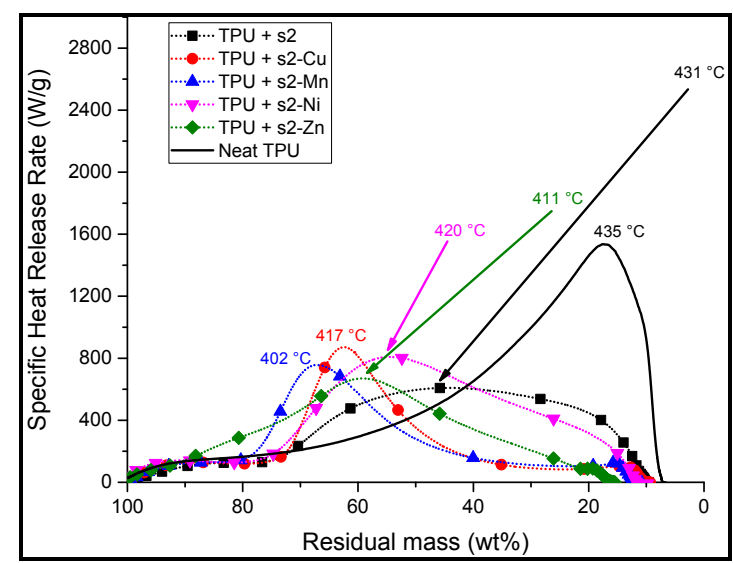

(b)

Figure 9. HRR vs. mass loss curves of (a) s1 and its corresponding complexes and (b) s2 and its corresponding complexes.

Except for that of TPU/s1-Zn, the HRR/mass-loss curves of TPU/s1 formulations (Figure 9a) show an increase in HRR starting at around $60 \%$ of residual mass. The HRR/mass-loss curve of the formulation containing TPU and s1 (black square, Figure 9a) has a much higher peak HRR than neat TPU. This suggests that most of the heat is released towards the end of the degradation of the samples and that there is a greater amount of combustibles released in TPU/s1 compared to neat TPU. The HRR/mass-loss curve of TPU/s1-Ni (magenta down-pointing triangle, Figure 9a) sample shows a slightly broader peak than that of neat TPU. However, the overall shape of the curve is very similar to the neat TPU curve, suggesting that the degradation of these formulations is somewhat similar in terms of HRR. The HRR/mass-loss curve of TPU/s1-Cu (red) shows a much broader range over which HRR is relatively low. This suggests that the pyrolysis of the TPU/s1-Cu releases combustible materials over a wider range of its mass during its degradation, but also at a smaller rate. The curve corresponding to TPU/s1-Zn shows a very different shape when compared to the others. Most of the heat is released at the beginning of its decomposition. After that, there is a relatively slow HRR until about $15 \%$ residual mass.

The curves corresponding to TPU/s2-M (Figure 9b) formulations show varied results. The HRR/mass-loss curves of most of the TPU/s2-M show that they start to significantly release heat at around $85-80 \mathrm{wt} \%$. However, it is interesting to note that there seems to be a preliminary phase between 0 and $20 \%$ weight loss whereby there is a constant heat release rate of approximately $100-150 \mathrm{~W} / \mathrm{g}$. This suggests that the early decomposition products of these formulations release non-combustible or hardly combustible products at the beginning of the pyrolysis. In the case of neat TPU, there is a slow and continuous increase of the HRR until the peak HRR is reached when the remaining mass is $17 \mathrm{wt} \%$. Once again, the curve corresponding to TPU/s2-Cu (red, filled circle, dotted) formulation shows a very broad peak with a relatively low peak HRR.

From the combination of HRR/Temperature and HRR/mass-loss curves, it can be observed that TPU/s1-Cu and TPU/s2-Cu release low-combustibility combustibles over a wide range of residual mass.

The TPU/s1-Zn formulation exhibits an intriguing behavior in the sense that most of its combustibles are released at a very early stage of its pyrolysis. Moreover, it has shown a relatively low peak heat release rate as compared to other s1-based formulations.

Finally, formulations of TPU and salen derivatives have shown that it can bring about a decrease in the HRR of the formulations by modifying the degradation steps of the formulations. This means that there would be a lower amount of combustibles released in a fire scenario. This suggests that these additives may have some fire retardant properties when they are incorporated in TPU. 


\subsection{Fire Properties Mass Loss Cone Calorimetry (MLC) Test}

In order to investigate the reaction to fire of the formulations, a mass loss cone calorimetry (MLC) test with a heat flux of $35 \mathrm{~kW} / \mathrm{m}^{2}$ was performed on the formulated samples to simulate a fire scenario. The HRR curves are presented on Figure 10.

The samples show similar behaviors right after ignition. There is a relatively steep increase in HRR at the beginning. A swelling of the sample is also observed in most cases. This swelling leads to the formation of a carbonaceous layer (char) with different thermal and mechanical robustness (visual observation and manual testing). Indeed, in the case of a thermally and mechanically stable char, the later can slow down the decomposition of the polymeric material lying under it. This can eventually limit the release inflammable gases, causing a lower HRR [14].

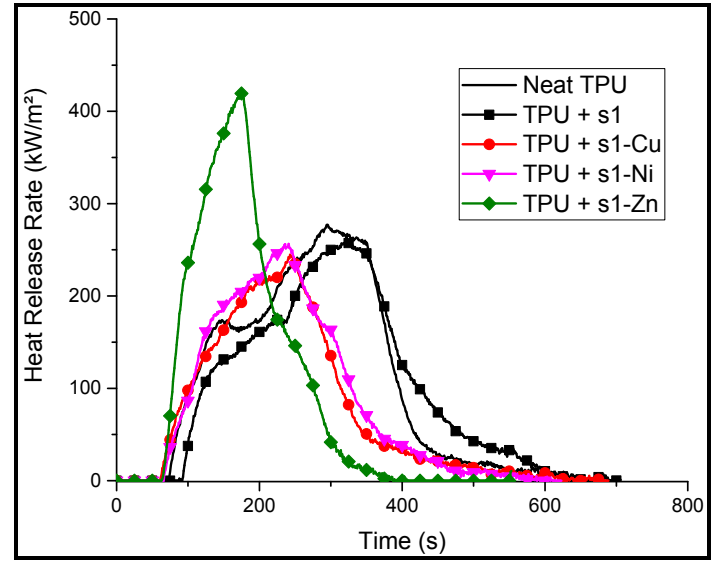

(a)

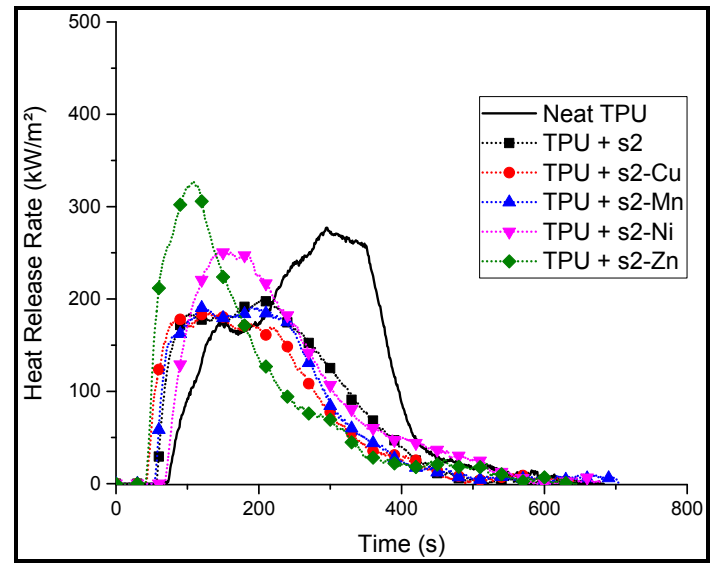

(b)

Figure 10. Mass loss cone calorimetry (MLC) curves of neat TPU, TPU/s1-M complexes (a) and TPU / s2-M complexes $(\mathbf{b})$ on samples $\left(50 \times 50 \times 3 \mathrm{~mm}^{3}\right)$ exposed to $35 \mathrm{~kW} / \mathrm{m}^{2}$.

With the exception of $\mathrm{Zn}$ based formulations, the HRR curves of all the samples showed a decrease in the pHRR compared to neat TPU. The data are summarized in Table 7.

The HRR plot of neat TPU (solid line) shows two pHRR and a total heat released of $66.7 \mathrm{MJ} / \mathrm{m}^{2}$. It shows two HRR peaks. The first step starts at the ignition whereby there is a steep increase in HRR. This increase peaks at around $176 \mathrm{~kW} / \mathrm{m}^{2}$ and $147 \mathrm{~s}$. After this, a slight decrease in HRR is observed until around $175 \mathrm{~s}\left(277 \mathrm{~kW} / \mathrm{m}^{2}\right)$. This is assigned to the formation of a char that protects the unburnt layer of the material and delays its decomposition. However, at around $200 \mathrm{~s}$, another sharp increase in the HRR is observed. This suggests that the inflammable decomposition gases are eventually released despite the formation of the char. The peak HRR is reached at this step (at around $300 \mathrm{~s}$ ) and corresponds to a heat release rate of $277 \mathrm{~kW} / \mathrm{m}^{2}$. The resulting residue is shown on Figure 11.

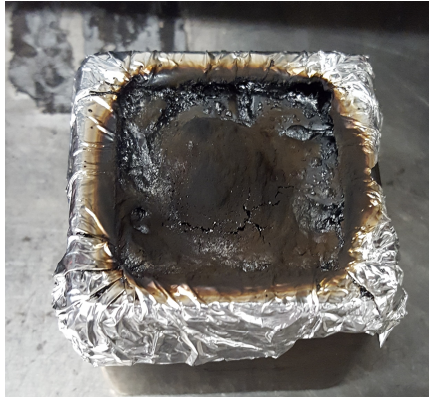

(a)

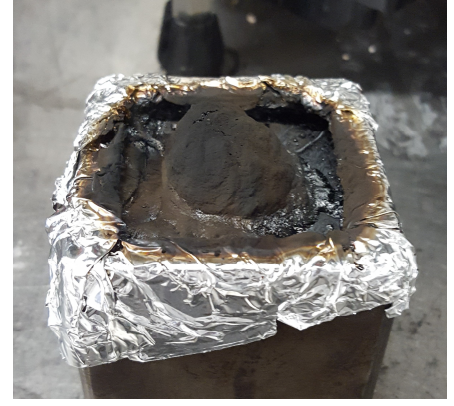

(b)

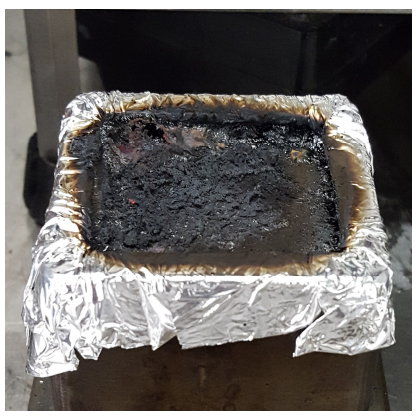

(c)

Figure 11. Residues of neat TPU (a); TPU/s1 (b) and TPU/s1-Cu (c). 
In the case of TPU/s1, the HRR curve shows no significant change brought about in the HRR values, neither in terms of pHRR nor in terms of THR.

TPU/s1-Cu and TPU/s1-Ni behave differently as compared to neat TPU and TPU/s1. HRR curves exhibit only one peak occurring before that of neat TPU ( $250 \mathrm{~s}$ vs. $350 \mathrm{~s})$ but the value of pHRR is not significantly decreased compared to neat TPU $(-1.8 \%)$. A potential explanation for this behavior is that the char has a relatively low thermomechanical strength, causing it to break or not form at all. This can be observed in the photo of TPU/s1-Cu (Figure 11c). However, these formulations have a significantly lower total heat released (THR) as compared to neat TPU ( $-27.3 \%$ for $\mathrm{Cu}$ and $-22.2 \%$ for $\mathrm{Ni}$ ).

MLC results for TPU/s1-Zn formulation showed a very high pHRR $\left(420 \mathrm{~kW} / \mathrm{m}^{2}\right)$ as compared to neat TPU (+51\%). This may correlate to the TGA of TPU/s1-Zn, which showed a very drastic decrease in mass at a relatively low temperature $\left(290^{\circ} \mathrm{C}\right)$, therefore causing the rapid release of combustibles early on during its decomposition under the radiative flux. A relatively sharp peak is observed right after ignition. It suggests that the sample degraded very quickly, releasing a lot of heat, and then forms a protective layer, which limited the release of energy. Evidence of the char formation of TPU/s1-Zn can be seen in the picture taken after the MLC test (Figure 12). However, the char being relatively fragile, it did not keep its shape and collapses. The high pHRR is assigned to the possibility that the presence of zinc catalyzed the degradation process of the formulation (as seen in the TGA), causing a rapid burning of the sample.

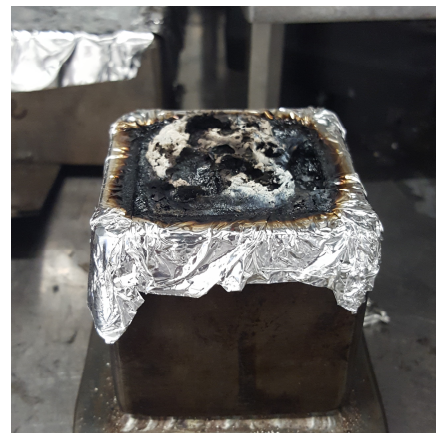

(a)

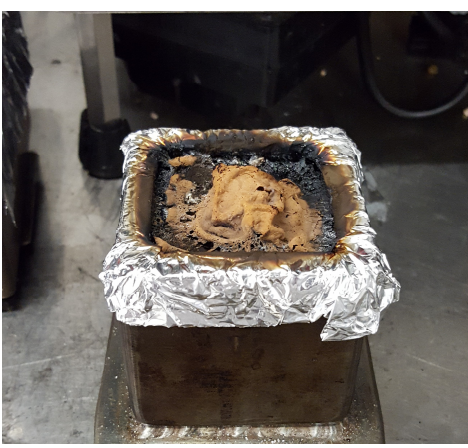

(b)

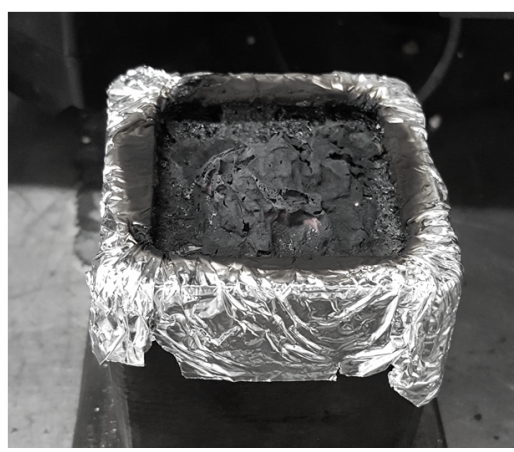

(c)

Figure 12. Samples of TPU/s1-Zn (a), TPU/s2-Mn (b) and TPU/s2-Cu (c) after the MLC test.

Formulations of $\mathbf{s} 2$ and its complexes exhibit a lower pHRR and THR as compared to its $\mathbf{s 1}$ counterparts. Indeed, the HRR curve of TPU/s2 itself shows a pHRR of $200 \mathrm{~kW} / \mathrm{m}^{2}$, which is about $28 \%$ lower than that of neat TPU and $23 \%$ lower than that of TPU/s1. This could be correlated to the fact that $\mathbf{s} \mathbf{2}$ has the ability to form thermally stable cross-linked charred structure (polyphenol) at high temperatures.

The HRR as a function of a time curve of neat TPU, s2 and $\mathbf{~ s 2 - M ~ f o r m u l a t i o n s ~ a r e ~ r e l a t i v e l y ~ s i m i l a r ~}$ immediately after ignition. There is the formation of a char, which inhibits the decomposition of the

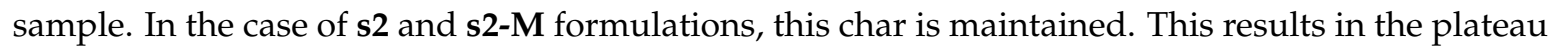
that is observed in the HRR curves of TPU/s2-Cu and TPU/s2-Mn. This also explains the lower pHRR of these samples (pHRR $<200 \mathrm{~kW} / \mathrm{m}^{2}$, as compared to neat TPU with a pHRR at $277 \mathrm{~kW} / \mathrm{m}^{2}$ ). Indeed, the shapes of the HRR curves of these two formulations are consistent with HRR curves of materials that exhibit charring when they are burnt [14]. These observations suggest that the cross-linking ability of $\mathbf{s} 2$ may have been maintained even if the ligand is complexed with $\mathrm{Cu}^{2+}$ or $\mathrm{Mn}^{2+}$.

As with TPU/s1-Zn, the HRR curve of TPU/s2-Zn shows a higher pHRR compared to neat TPU $(+17.7 \%)$. However, the $\mathbf{s} 2-\mathbf{Z n}$ formulation induces a lower pHRR than TPU/s1-Zn. This may be due to the ability of $\mathbf{s} \mathbf{2}$ to form a cross-linked polyphenolic structure in the polymer matrix, forming a protective thermally stable layer, thus decreasing the pHRR. The zinc cation may catalyze the 
formation of the cross-linked structure. These bond formations being exothermic, it could explain the exceedingly high $\mathrm{pHRR}$ as compared to neat TPU.

The HRR curves of TPU/s2-Cu and TPU/s2-Mn show the lowest pHRR and THR of all of the formulations. The values are similiar and are consistent with PCFC results. Indeed, when compared to neat TPU, they showed a relatively low pHRR both in the PCFC test $(-56 \%$ for $\mathrm{Cu}$ and $-51 \%$ for $\mathrm{Mn}$ as compared to neat TPU) and in the MLC test ( $-32.5 \%$ for $\mathrm{Cu}$ and $-30.7 \%$ for Mn). These results have shown that there is no distinct correlation between MLC and PCFC results. Indeed, considering that after the PCFC tests, the most promising sample as a fire retardant was the TPU/s1-Zn formulation. However, upon performing MLC, it was seen that, among all of the samples tested, TPU/s1-Zn has the highest $\mathrm{pHRR}$, making it a mediocre candidate as a FR.

Table 7. Summary of the results for the MLC test $\left(50 \times 50 \times 3 \mathrm{~mm}^{3}\right)$.

\begin{tabular}{ccc}
\hline Sample & pHRR $\left(\mathbf{k W} / \mathbf{m}^{\mathbf{2}}\right)$ & THR $\left.\mathbf{( M J} / \mathbf{m}^{\mathbf{2}}\right)$ \\
\hline Neat TPU & 277 & 66.7 \\
TPU + s1 & $260(-6 \%)$ & $65.5(-2 \%)$ \\
TPU + s1-Cu & $244(-11 \%)$ & $48.5(-27 \%)$ \\
TPU + s1-Ni & $256(-7 \%)$ & $51.9(-22 \%)$ \\
TPU + s1-Zn & $420(+51 \%)$ & $54.3(-18 \%)$ \\
\hline TPU + s2 & $200(-27 \%)$ & $50.5(-24 \%)$ \\
TPU + s2-Cu & $187(-32 \%)$ & $45.0(-32 \%)$ \\
TPU + s2-Mn & $192(-30 \%)$ & $47.3(-29 \%)$ \\
TPU + s2-Ni & $251(-9 \%)$ & $54.9(-17 \%)$ \\
TPU + s2-Zn & $326(+17 \%)$ & $52.4(-21 \%)$ \\
\hline
\end{tabular}

In conclusion, some of the formulated samples have shown that they exhibit enhanced reaction to fire evidenced by their lower pHRR as compared to neat TPU. On the other hand, other formulations have shown that they have a total heat released that is significantly lower than that of neat TPU. With the exception of TPU/s1, all of the formulations showed a THR, which was at least 15\% lower than that of neat TPU.

\section{Materials and Methods}

\subsection{Instrumental Analyses}

\subsubsection{Thermogravimetric Analysis}

Thermogravimetric analyses (TGA) were conducted on a Setaram apparatus model TG 92-16 (Caluire-et-Cuire, France). Samples of 6-10 mg were placed in open alumina pans and heated either under nitrogen atmosphere or in air with a heating rate of $10^{\circ} \mathrm{C} / \mathrm{min}$.

\subsubsection{Pyrolysis Combustion Flow Calorimetry}

Fire performance was evaluated based on heat release rate (HRR) curve obtained from an FAA Micro calorimeter (FAA Fire testing technology, East Grinstead, UK) operated at $1{ }^{\circ} \mathrm{C} / \mathrm{s}$ to $750{ }^{\circ} \mathrm{C}$ in the pyrolysis zone according to ASTM D7309 method A. The combustion zone was set at $900{ }^{\circ} \mathrm{C}$. Oxygen and nitrogen flow rates were set at 20 and $80 \mathrm{~mL} / \mathrm{min}$, respectively. Repeatability is confirmed by three consecutive trials (error margin $\pm 10 \%$ ).

The results obtained were corrected after conducting a TGA under nitrogen atmosphere of each sample. The conditions of the TGA were the same as that of the PCFC $\left(1{ }^{\circ} \mathrm{C} / \mathrm{s}\right.$ to $750{ }^{\circ} \mathrm{C}$, under nitrogen atmosphere). The residual mass at a given temperature allowed the calculation of the specific heat release rate at any given temperature. 


\subsubsection{Mass Loss Cone Calorimeter}

The mass loss cone calorimeter (Fire testing technology (FTT), Fire testing technology, East Grinstead, UK) is used for recording HRR curve. Plates $\left(50 \times 50 \times 3 \mathrm{~mm}^{3}\right.$ plates $)$ for cone calorimeter test were made via compression molding using a Darragon press apparatus (Pinette P.E.I, Chalon sur Saône, France). Plates were wrapped in aluminum foil leaving the upper surface exposed to the heater and placed in a horizontal position on a ceramic block encased in a metallic container at a distance of $35 \mathrm{~mm}$ from the cone base. An external heat flux of $35 \mathrm{~kW} / \mathrm{m}^{2}$ was used for all of the experiments.

\subsection{Synthesis and Characterisation}

Salen and hydroxysalens s1 and s2 were obtained using a conventional procedure for salen synthesis based on the condensation of salicylaldehyde or its hydroxy-derivatives with diamine [6] Most salen complexes were synthesized according to procedure described in [5]. Zinc-salen complexes were prepared following the procedure described in [8].

Reagents were purchased from Aldrich (Sigma-Aldrich Chimie S.a.r.l., Lyon, France) or Lancaster Synthesis (Alfa Aesar, Haverhill, MA, USA) and were used without further purification. Diethyl ether, methanol and absolute ethanol were reagent grade commercial solvents and were used without further purification.

NMR spectra were recorded on a Bruker Avance 300 spectrometer (Billerica, MA, USA). Chemical shifts $(\delta)$ are referenced to internal solvent and given in ppm. Coupling constants $(J)$ are given in Hz. The following abbreviations apply to spin multiplicity: s (singlet), d (doublet), $\mathrm{t}$ (triplet), q (quartet), $\mathrm{m}$ (multiplet) and bs (broad singlet).

\subsection{1. $N, N^{\prime}$-Bis(salicylidene)ethylenediamine (s1)}

To a solution of ethylenediamine $(5.34 \mathrm{~mL} ; 4.8 \mathrm{~g} ; 0.08 \mathrm{~mol})$ in $60 \mathrm{~mL}$ of absolute ethanol, salicylaldehyde $(16.7 \mathrm{~mL} ; 19.5 \mathrm{~g} ; 0.16 \mathrm{~mol})$ in $80 \mathrm{~mL}$ of ethanol was added dropwise with vigorous stirring. The product precipitated immediately and the mixture was refluxed for two hours, and then kept at room temperature and filtered. The product recrystallized from EtOH was filtered, washed with cold ethanol and ethyl ether, and then dried at $80^{\circ} \mathrm{C}$ to give the desired Schiff base s1 as yellow crystals, $20.2 \mathrm{~g}(94 \%)$. ${ }^{1} \mathrm{H}-\mathrm{NMR}$ (DMSO-d $)$ ) 3.92 (s, $\left.4 \mathrm{H}\right), 6.81-6.95$ (m, $\left.4 \mathrm{H}\right), 7.35$ (m, $\left.4 \mathrm{H}\right), 8.57$ (s, $\left.2 \mathrm{H}\right)$; ${ }^{13} \mathrm{C}-\mathrm{NMR}\left(\mathrm{DMSO}-d_{6}\right) \delta 58.8\left(\mathrm{CH}_{2}\right), 116.4\left(\mathrm{C}_{\mathrm{Ar}}\right), 118.5\left(\mathrm{C}_{\mathrm{Ar}}\right), 118.6\left(\mathrm{C}_{\mathrm{Ar}}\right), 131.6\left(\mathrm{C}_{\mathrm{Ar}}\right), 132.3\left(\mathrm{C}_{\mathrm{Ar}}\right)$, $160.4\left(\mathrm{C}_{\mathrm{Ar}}\right), 166.8(\mathrm{CN})$. MS (NanoESI) $m / z 269.1(\mathrm{M}+\mathrm{H})^{+} . \mathrm{Mp}=130{ }^{\circ} \mathrm{C}$.

\subsection{2. $N, N^{\prime}$-Bis(4-hydroxysalicylidene)ethylenediamine (s2)}

According to the method described for s1, $21.9 \mathrm{~g}(0.16 \mathrm{~mol})$ of 2,4-dihydroxybenzaldehyde and $5.34 \mathrm{~mL}(4.8 \mathrm{~g}, 0.08 \mathrm{~mol})$ of ethylenediamine yielded compound s2 $(28.1 \mathrm{~g} ; 94 \%)$ orange solid; ${ }^{1} \mathrm{H}-\mathrm{NMR}$ $\left(\mathrm{DMSO}-d_{6}\right) \delta 3.77(\mathrm{~s}, 4 \mathrm{H}), 6.15(\mathrm{~d}, J=2.4 \mathrm{~Hz}, 2 \mathrm{H}), 6.26(\mathrm{dd}, J=2.4,8.6 \mathrm{~Hz}, 2 \mathrm{H}), 7.16(\mathrm{~d}, J=8.6 \mathrm{~Hz}$, $2 \mathrm{H}), 8.35(\mathrm{~s}, 2 \mathrm{H}) ;{ }^{13} \mathrm{C}-\mathrm{NMR}\left(\mathrm{DMSO}-d_{6}\right) \delta 57.8\left(\mathrm{CH}_{2}\right), 102.5\left(\mathrm{C}_{\mathrm{Ar}}\right), 106.9\left(\mathrm{C}_{\mathrm{Ar}}\right), 111.2\left(\mathrm{C}_{\mathrm{Ar}}\right), 133.4\left(\mathrm{C}_{\mathrm{Ar}}\right)$, $161.7\left(\mathrm{C}_{\mathrm{Ar}}\right), 164.3\left(\mathrm{C}_{\mathrm{Ar}}\right), 165.7(\mathrm{CN})$. MS (NanoESI) $\mathrm{m} / z 301.2(\mathrm{M}+\mathrm{H})^{+}$. Mp $>190{ }^{\circ} \mathrm{C}$ (decomposition).

\subsection{3. $N, N^{\prime}$-Bis(salicylidene)ethylenediamine Copper (II) Complex, s1-Cu}

$N, N^{\prime}$-bis(salicylidene)ethylenediamine s1 $(8.1 \mathrm{~g}, 0.03 \mathrm{~mol})$ was dissolved in $70 \mathrm{~mL}$ absolute ethanol; then, a solution of copper (II) acetate $(6.0 \mathrm{~g}, 0.03 \mathrm{~mol})$ in $40 \mathrm{~mL}$ of water was added dropwise and the mixture was refluxed under vigorous stirring for $2.5 \mathrm{~h}$. The green precipitate was collected by filtration, washed thoroughly with ethanol and then dried at $80^{\circ} \mathrm{C}$ to give $N, N^{\prime}$-bis(salicylidene)ethylenediamine copper (II) complex s1-Cu (6.96 g, 70\%) as a fine green powder. NMR spectra of the complex was not recorded due to paramagnetism of $\mathrm{Cu}$ (II) (this well-known phenomenon leads to poor resolution of the spectra). MS (NanoESI) $m / z 330.0(\mathrm{M}+\mathrm{H})^{+}, 659.1(\text { dimer }+\mathrm{H})^{+} . \mathrm{Mp}>260{ }^{\circ} \mathrm{C}$. 


\subsection{4. $N, N^{\prime}$-Bis(salicylidene)ethylenediamine Nickel (II) Complex, s1-Ni}

$\mathrm{N}, \mathrm{N}^{\prime}$-bis(salicylidene)ethylenediamine $\mathbf{s 1}(6.01 \mathrm{~g}, 0.022 \mathrm{~mol})$ was dissolved in $70 \mathrm{~mL}$ absolute ethanol, then a solution of nickel (II) acetate $(5.51 \mathrm{~g}, 0.022 \mathrm{~mol})$ in $40 \mathrm{~mL}$ of water was added dropwise and the mixture was refluxed under vigorous stirring for $2.5 \mathrm{~h}$. The orange precipitate was collected by filtration, washed thoroughly with ethanol, and then dried at $80{ }^{\circ} \mathrm{C}$ to give $N, N^{\prime}$-bis(salicylidene)ethylenediamine Nickel (II) complex s1-Ni $(5.87 \mathrm{~g}, 80.6 \%)$ as an orange powder. ${ }^{1} \mathrm{H}-\mathrm{NMR}\left(300 \mathrm{MHz}\right.$, DMSO- $\left.d_{6}\right) \delta$ ppm 3.29-3.35 (m, $\left.1 \mathrm{H}\right) 3.42(\mathrm{~s}, 4 \mathrm{H}) 6.51(\mathrm{t}, J=7.25 \mathrm{~Hz}, 2 \mathrm{H}) 6.70(\mathrm{~d}$, $J=8.67 \mathrm{~Hz}, 2 \mathrm{H}) 7.17(\mathrm{ddd}, J=8.43,6.92,1.79 \mathrm{~Hz}, 2 \mathrm{H}) 7.25(\mathrm{dd}, J=7.82,1.60 \mathrm{~Hz}, 2 \mathrm{H}) 7.89(\mathrm{~s}, 2 \mathrm{H})$. ${ }^{13} \mathrm{C}-\mathrm{NMR}$ (75 MHz, DMSO- $d_{6}$ ) $\delta$ ppm 58.45 (s, $\left.1 \mathrm{C}\right) 114.75$ (s, $\left.1 \mathrm{C}\right) 120.22$ (s, $\left.1 \mathrm{C}\right) 120.79$ (s, $1 \mathrm{C}$ ) 133.22 (s, 1 C) 133.98 (s, 1 C) 163.07 (s, 1 C) 164.40 (s, 1 C). MS (NanoESI) $\mathrm{m} / z 324.0 \mathrm{M}^{+}$. Mp > $260{ }^{\circ} \mathrm{C}$.

\subsection{5. $N, N^{\prime}$-Bis(salicylidene)ethylenediamine Zinc (II) Complex, s1-Zn}

The complex s1-Zn was prepared by first treating salicylaldehyde $(2.15 \mathrm{~mL}, 0.02 \mathrm{~mol})$ with zinc (II) acetate $(2.26 \mathrm{~g}, 0.01 \mathrm{~mol})$ in methanol $(100 \mathrm{~mL})$ for $30 \mathrm{~min}$ at room temperature. Then, ethylenediamine $(0.68 \mathrm{~mL}, 0.01 \mathrm{~mol})$ was added to the solution, and stirring was maintained at room temperature overnight. During that time, a light yellow precipitate formed. The precipitate was collected by filtration, washed with cold methanol and ether, and dried at $80{ }^{\circ} \mathrm{C}$, affording $3.00 \mathrm{~g}, 80 \% .{ }^{1} \mathrm{H}-\mathrm{NMR}$ $\left(300 \mathrm{MHz}, \mathrm{DMSO}-d_{6}\right) \delta \mathrm{ppm} 3.72(\mathrm{~s}, 4 \mathrm{H}) 3.92(\mathrm{~s}, 1 \mathrm{H}) 6.34-6.50(\mathrm{~m}, 2 \mathrm{H}) 6.55-6.68(\mathrm{~m}, 1 \mathrm{H})$ $6.61(\mathrm{~d}, J=8.29 \mathrm{~Hz}, 1 \mathrm{H}) 7.09-7.44(\mathrm{~m}, 4 \mathrm{H}) 8.43(\mathrm{~s}, 2 \mathrm{H}) 13.34-13.45(\mathrm{~m}, 1 \mathrm{H}) .{ }^{13} \mathrm{C}-\mathrm{NMR}(75 \mathrm{MHz}$, DMSO- $\left.d_{6}\right) \delta$ ppm 55.75 (s, 1 C) 112.15 (s, 1 C) $119.31(\mathrm{~s}, 1 \mathrm{C}) 122.68$ (s, 1 C) 132.77 (s, 1 C) 134.73 (s, $\left.1 \mathrm{C}\right)$ $167.97(\mathrm{~s}, 1 \mathrm{C}) 171.01(\mathrm{~s}, 1 \mathrm{C})$. MS (NanoESI) $\mathrm{m} / z 331.0(\mathrm{M}+\mathrm{H})^{+} . \mathrm{Mp}>260^{\circ} \mathrm{C}$.

\subsection{6. $\mathrm{N}, \mathrm{N}^{\prime}$-Bis(4-hydroxysalicylidene)ethylenediamine Copper (II) Complex, s2-Cu}

$\mathrm{N}, \mathrm{N}^{\prime}$-bis(4-hydroxysalicylidene)ethylenediamine s2 $(9.0 \mathrm{~g}, 0.03 \mathrm{~mol})$ was dissolved in $70 \mathrm{~mL}$ absolute ethanol, then a solution of copper (II) acetate $(6.0 \mathrm{~g}, 0.03 \mathrm{~mol})$ in $40 \mathrm{~mL}$ of water was added dropwise and the mixture was refluxed under vigorous stirring for $2.5 \mathrm{~h}$. The purple precipitate was collected by filtration, washed thoroughly with ethanol, and then dried at $80{ }^{\circ} \mathrm{C}$ to give $N, N^{\prime}$-bis(4-hydroxysalicylidene)ethylenediamine copper (II) complex s1-Cu (9.7 g, 89\%) as a purple powder. NMR spectra of the complex was not recorded due to paramagnetism of $\mathrm{Cu}$ (II) (this well-known phenomenon leads to poor resolution of the spectra). MS (NanoESI) $\mathrm{m} / \mathrm{z}$ $362.0(\mathrm{M}+\mathrm{H})^{+} . \mathrm{Mp}>260^{\circ} \mathrm{C}$.

\subsection{7. $N, N^{\prime}$-Bis(4-hydroxysalicylidene)ethylenediamine Manganese (II) Complex, s2-Mn}

$N, N^{\prime}$-bis(4-hydroxysalicylidene)ethylenediamine s2 (3.60 g, $\left.0.012 \mathrm{~mol}\right)$ was dissolved in $70 \mathrm{~mL}$ absolute ethanol, then a solution of manganese (II) acetate $(2.94 \mathrm{~g}, 0.012 \mathrm{~mol})$ in $40 \mathrm{~mL}$ of water was added dropwise and the mixture was refluxed under vigorous stirring for $2.5 \mathrm{~h}$. The brown precipitate was collected by filtration, washed thoroughly with ethanol, and then dried at $80^{\circ} \mathrm{C}$ to give $N, N^{\prime}$-bis(4-hydroxysalicylidene)ethylenediamine manganese (II) complex s1-Mn (3.1 g, 73\%) as a brown solid. NMR spectra of the complex was not recorded due to paramagnetism of Mn(II) (this well-known phenomenon leads to poor resolution of the spectra). MS (NanoESI) $m / z 353.0(\mathbf{M})^{+}$. $\mathrm{Mp}>260^{\circ} \mathrm{C}$.

\subsection{8. $N, N^{\prime}$-Bis(4-hydroxysalicylidene)ethylenediamine Manganese (II) Complex, s2-Ni}

$N, N^{\prime}$-bis(4-hydroxysalicylidene)ethylenediamine s2 $(7.00 \mathrm{~g}, 0.022 \mathrm{~mol})$ was dissolved in $50 \mathrm{~mL}$ absolute ethanol, then a solution of nickel (II) acetate $(5.51 \mathrm{~g}, 0.022 \mathrm{~mol})$ in $50 \mathrm{~mL}$ of ethanol was added dropwise, and the mixture was refluxed under vigorous stirring for 2 . The red precipitate was collected by filtration, washed thoroughly with ethanol, and then dried at $80{ }^{\circ} \mathrm{C}$ to give $\mathrm{N}, \mathrm{N}^{\prime}$-bis(4-hydroxysalicylidene)ethylenediamine cobalt (II) complex s2-Ni (5.36 g, 89\%) as a red powder. ${ }^{1} \mathrm{H}-\mathrm{NMR}\left(300 \mathrm{MHz}, \mathrm{DMSO}-d_{6}\right) \delta$ ppm 3.25-3.31 (m, $\left.2 \mathrm{H}\right) 6.04(\mathrm{~d}, J=8.69 \mathrm{~Hz}, 2 \mathrm{H}) 6.05(\mathrm{~s}, 1 \mathrm{H})$ 
$7.05(\mathrm{~d}, J=8.10 \mathrm{~Hz}, 1 \mathrm{H}) 7.62(\mathrm{~s}, 1 \mathrm{H}) 9.73(\mathrm{br} \mathrm{s}, 1 \mathrm{H}) .{ }^{13} \mathrm{C}-\mathrm{NMR}\left(75 \mathrm{MHz}\right.$, DMSO- $\left.d_{6}\right) \delta \mathrm{ppm} 58(\mathrm{~s}, 1 \mathrm{C})$ 104 (s, 1 C) 106 (s, 1 C) 115 (s, 1 C) 135 (s, 1 C) 161 (s, 1 C) 163 (s, 1 C) 166 (s, 1 C). MS (NanoESI) m/z $356.0(\mathrm{M})^{+} . \mathrm{Mp}>260^{\circ} \mathrm{C}$.

\subsection{9. $N, N^{\prime}$-bis(4-hydroxysalicylidene)ethylenediamine Manganese (II) Complex, s2-Zn}

The complex s2-Zn was prepared by first treating 4-hydroxysalicylaldehyde ( $2.82 \mathrm{~g}, 0.020 \mathrm{~mol})$ with $\mathrm{Zn}(\mathrm{OAc})_{2} \cdot 2 \mathrm{H}_{2} \mathrm{O}(2.26 \mathrm{~g}, 0.010 \mathrm{~mol})$ in methanol $(70 \mathrm{~mL})$ for $30 \mathrm{~min}$ at room temperature. Then, ethylenediamine $(0.68 \mathrm{~mL}, 0.01 \mathrm{~mol})$ in $35 \mathrm{~mL}$ of methanol was added to the solution, and stirring was maintained at room temperature overnight. During that time, a yellow precipitate formed. The precipitate was collected by filtration, washed with cold methanol and ether, and dried under vacuum, affording $3.23 \mathrm{~g}, 87 \% .{ }^{1} \mathrm{H}-\mathrm{NMR}\left(300 \mathrm{MHz}, \mathrm{DMSO}-d_{6}\right) \delta \mathrm{ppm} 3.59(\mathrm{~s}, 2 \mathrm{H}) 5.89-5.96(\mathrm{~m}, 1 \mathrm{H})$ $5.90-5.95(\mathrm{~m}, 1 \mathrm{H}) 5.95(\mathrm{~s}, 1 \mathrm{H}) 5.98(\mathrm{~s}, 1 \mathrm{H}) 6.92(\mathrm{~d}, J=8.48 \mathrm{~Hz}, 1 \mathrm{H}) 8.21(\mathrm{~s}, 1 \mathrm{H}) 9.39$ (br s, $1 \mathrm{H})$. ${ }^{13} \mathrm{C}-\mathrm{NMR}\left(75 \mathrm{MHz}, \mathrm{DMSO}-d_{6}\right) \delta$ ppm 56 (s, 2 C) 103 (1 C) 107 (1 C) 113 (1 C) 136 (1 C) 162 (1 C) 167 (1 C) 173 (1 C). MS (NanoESI) $\mathrm{m} / z 363.1(\mathrm{M}+\mathrm{H})^{+}$. Mp $>260^{\circ} \mathrm{C}$.

\subsection{Formulations, Processing and Sampling of Polymer}

Thermoplastic Polyurethane (TPU) C85A (polyester polyurethane) was kindly supplied by BASF (Ludwigshafen, Germany).

TPU is dried for $12 \mathrm{~h}$ at $70{ }^{\circ} \mathrm{C}$ before use. Compounding of formulations with a ratio of $10: 1 \mathrm{wt} \%$ (TPU:additive) of salen and their complexes was performed using a Microextruder (DSM Micro15, Xplore Instruments BV, Sittard, The Netherlands) under a nitrogen atmosphere at $190{ }^{\circ} \mathrm{C}(50 \mathrm{rpm}$ for 5 min of mixing). The formulation was ground in liquid nitrogen in an ultracentrifuge to produce a powder and dried at $80^{\circ} \mathrm{C}$ for $6 \mathrm{~h}$ before use.

\section{Conclusions}

This work was devoted to the synthesis of some metallosalen and metallosalen $(\mathrm{OH})_{2}$ with the aim of testing them as new FR in thermoplastic polyurethane and comparing them with the copper-salen complex, which has already been studied before. Seven metallosalens were successfully synthesized using simple chemistry in one or two-step reactions using readily available low-impact reactants (ethanol, salen and earth abundant metals). These substances were easily incorporated in TPU. Relatively low loadings with 10:1 weight ratios of TPU/FR were used. These formulations have shown promise in the field of FR. Moreover, MLC results are promising in the sense that the total heat released by the formulations are significantly lower than that of neat TPU. PCFC results of the formulation have also shown promising results with a decrease in HRR as low as 50- in the case of TPU/s2-Cu or TPU/s2-Mn and a decrease in THR of 40-60\%. This confirms that this class of flame-retardants has a lot of potential in the field of polymer chemistry. By extension, the fire properties of the TPU/metallosalen complexes can be explained by the cross-linking ability of s2 under thermal stress. However, as the metallosalens have an enhanced thermal stability, their applicability may be extended to engineering polymers with higher processing temperatures.

Acknowledgments: This work has received funding from the European Research Council (ERC) under the European Union's H2020 - the Framework Programme for Research and Innovation (2014-2020) ERC Grant Advances Agreement N 670747-ERC 2014 AdG/FireBar-Concept for FireBar Concept project.

Author Contributions: Christophe Penverne has carried out and analyzed the mass spectra of all of the synthesized additives that were used in this work. Gaëlle Fontaine and Serge Bourbigot supervised the work and contributed to the paper writing. Aditya Ramgobin carried out the synthesis and characterization by NMR of the samples. He also performed and analyzed all of the thermal and flame tests. He performed the scientific discussion and conclusions and wrote the paper.

Conflicts of Interest: The authors declare no conflict of interest. 


\section{References}

1. Nelson, G.L. Fire and Polymers: An Overview. ACS Symp. Ser. 1995, 599, 1-26. [CrossRef]

2. Wilkie, C.A.; Morgan, A.B. Fire Retardancy of Polymeric Materials, 2nd ed.; Wilkie, C.A., Morgan, A.B., Eds.; CRC Press: Boca Raton, FL, USA, 2009.

3. Aschberger, K.; Campia, I.; Pesudo, L.Q.; Radovnikovic, A.; Reina, V. Chemical Alternatives Assessment of Different Flame Retardants-A Case Study Including Multi-Walled Carbon Nanotubes as Synergist. Environ. Int. 2017, 101, 27-45. [CrossRef] [PubMed]

4. Joseph, P.; Ebdon, J.R. Recent Developments in Flame-Retarding Thermoplastics and Thermosets. Fire Retard. Mater. 2001, 220-263. [CrossRef]

5. Schartel, B. Phosphorus-Based Flame Retardancy Mechanisms-Old Hat or a Starting Point for Future Development? Materials 2010, 3, 4710-4745. [CrossRef]

6. Cozzi, P.G. Metal-Salen Schiff Base Complexes in Catalysis: Practical Aspects. Chem. Soc. Rev. 2004, 33, 410-421. [CrossRef] [PubMed]

7. Verquin, G.; Fontaine, G.; Bria, M.; Zhilinskaya, E.; Abi-Aad, E.; Aboukaïs, A.; Baldeyrou, B.; Bailly, C.; Bernier, J.L. DNA Modification by oxovanadium(IV) Complexes of Salen Derivatives. J. Biol. Inorg. Chem. 2004, 9, 345-353. [CrossRef] [PubMed]

8. Jacobsen, E.N.; Zhang, W.; Muci, A.R.; Ecker, J.R.; Deng, L. Highly Enantioselective Epoxidation Catalysts Derived from 1,2-Diaminocyclohexane. J. Am. Chem. Soc. 1991, 113, 7063-7064. [CrossRef]

9. Fontaine, G.; Turf, T.; Bourbigot, S. Chapter 20 Salen Copper Complexes: A Novel Flame Retardant for Thermoplastic Polyurethane. ACS Symp. Ser. 2009, 329-340. [CrossRef]

10. Naik, A.D.; Fontaine, G.; Bellayer, S.; Bourbigot, S. Salen Based Schiff Bases to Flame Retard Thermoplastic Polyurethane Mimicking Operational Strategies of Thermosetting Resin. RSC Adv. 2015, 5, 48224-48235. [CrossRef]

11. Naik, A.D.; Fontaine, G.; Bellayer, S.; Bourbigot, S. Crossing the Traditional Boundaries: Salen-Based Schiff Bases for Thermal Protective Applications. ACS Appl. Mater. Interfaces 2015, 7, 21208-21217. [CrossRef] [PubMed]

12. Naik, A.D.; Fontaine, G.; Bellayer, S.; Touati, N.; Tayeb, K.B.; Vezin, H.; Bourbigot, S. Projection of coordination complexes as fire protective agents for thermoplastic polyurethane: Investigation by EPR spectroscopy. 2007; unpublished manuscript.

13. Albrecht, M.; Bedford, R.; Plietker, B. Catalytic and Organometallic Chemistry of Earth-Abundant Metals. Organometallics 2014, 33, 5619-5621. [CrossRef]

14. Lindholm, J.; Brink, A.; Hupa, M. Cone Calorimeter-A Tool for Measuring Heat Release Rate. In Finnish-Swedish Flame Days 2009; Åbo Akademi Process Chemistry Centre: Åbo, Finland, 2009. 